Boletín de la Sociedad Geológica Mexicana

VOLUMEN 68, NÚM. 2, 2016, P. 199-214

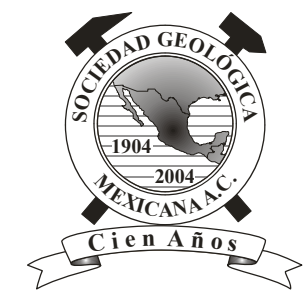

\title{
Acoplamiento sismogénico en la zona de subducción de Michoacán- Colima-Jalisco, México
}

\author{
M. Rosario Martínez-López ${ }^{1, *}$, Carlos Mendoza² \\ ${ }^{1}$ Posgrado en Ciencias de la Tierra, Universidad Nacional Autónoma de México, Apartado Postal 1-742, Querétaro, Qro. 76230, \\ México. \\ ${ }^{2}$ Centro de Geociencias, Universidad Nacional Autónoma de México, Apartado Postal 1-742, Querétaro, Qro. 76230, México. \\ *rosariomar55@hotmail.com
}

\begin{abstract}
Resumen
Se analizaron los hipocentros y mecanismos focales de los sismos ocurridos en el suroeste de México entre 1964 y 2008 para examinar el ancho sismogénico de la zona de subducción de Michoacán-Colima-Jalisco. Esta zona incluye el segmento de Michoacán en la porción noroeste de la placa de Cocos y el segmento de Jalisco que corresponde a la placa de Rivera. En el segmento de Michoacán la profundidad máxima estimada para la zona de acoplamiento sismogénico es $40 \mathrm{~km}$. Esta profundidad es mayor al valor de $25 \mathrm{~km}$ propuesto anteriormente para la placa de Cocos. Para la placa de Rivera la profundidad máxima del contacto sismogénico también es $40 \mathrm{~km}$, consistente con el valor propuesto anteriormente. Este valor sugiere un ancho sismogénico distinto para cada segmento por la diferencia en el ángulo de buzamiento entre las placas de Cocos y de Rivera. Las dimensiones del contacto sismogénico estimadas para el segmento de Michoacán (300 km x $95 \mathrm{~km})$ y para el segmento de Jalisco (420 km x $75 \mathrm{~km})$ indican una magnitud máxima similar de Mw 8.3 con un deslizamiento promedio esperado de $2 \mathrm{~m}$. Estos resultados tienen implicaciones importantes para la estimación del potencial sísmico en la zona de subducción de Michoacán-Colima-Jalisco.
\end{abstract}

Palabras clave: Sismicidad, mecanismos focales, subducción en México, zona sismogénica.

\begin{abstract}
Hypocenters and focal mechanisms of earthquakes in southwestern Mexico between 1964 and 2008 were analyzed to examine the seismogenic width of the Michoacan-Colima-Jalisco subduction zone. This subduction zone includes the Michoacan segment in the northwest portion of the Cocos plate and the Jalisco segment, which corresponds to the Rivera plate. In the Michoacan segment, the maximum depth of seismogenic coupling is approximately $40 \mathrm{~km}$. This depth is greater than the value of $25 \mathrm{~km}$ previously proposed for the entire Cocos plate. For the Rivera plate, the maximum seismogenic depth is also $40 \mathrm{~km}$, consistent with the previously proposed value. This value suggests a different seismogenic width for each segment due to the difference in dip between the Cocos and Rivera plates. The dimensions of the seismogenic contact estimated for the Michoacan segment (300 km $95 \mathrm{~km})$ and for the Jalisco segment $(420 \mathrm{~km} \times 75 \mathrm{~km})$ indicate a similar maximum magnitude of $M w 8.3$ with an expected average slip of $2 \mathrm{~m}$. These results have important implications for the estimation of the seismic potential in the Michoacan-Colima-Jalisco subduction zone.
\end{abstract}

Keywords: Seismicity, focal mechanisms, subduction in Mexico, seismogenic zone. 


\section{Introducción}

La sismicidad en las zonas de subducción es una de las principales fuentes de amenaza sísmica en el mundo. Estas regiones generan los sismos de mayor magnitud y por ello, generalmente los más destructivos al afectar centros urbanos. En el suroeste de México, la zona de subducción abarca desde la costa de Jalisco hasta la frontera con Guatemala a lo largo de la costa del Océano Pacífico. Esta zona de subducción incluye la región de Michoacán-Colima-Jalisco donde las placas oceánicas de Cocos y de Rivera subducen bajo la placa continental de Norteamérica (Nixon, 1982; DeMets y Stein, 1990; Pardo y Suárez, 1995). El sismo más grande registrado en México durante la época instrumental sismológica ocurrió en esta región en la costa de Jalisco el 3 de junio de 1932 (Eissler y McNally, 1984). Singh et al. (1984) estimaron una magnitud de ondas superficiales (Ms) de 8.2 para este sismo y sugieren que tuvo una ruptura compleja con base al análisis de sismogramas registrados en Europa a distancias mayores a $85^{\circ}$ en instrumentos tipo Wiechert (Uppsala, Gottingen y Copenhagen) y GalitzinWilip (Stuttgart). Singh et al. (1984) modelaron estos registros utilizando cuatro subeventos distintos con una duración total de aproximadamente $90 \mathrm{~s}$.

El evento del 3 de junio 1932 fue seguido por otro sismo de magnitud Ms 7.6 el 18 de junio al suroeste de Manzanillo (Eissler y McNally, 1984). Singh et al. (1985) estimaron una longitud de ruptura de $220 \mathrm{~km}$ para el evento del 3 de Junio y una longitud de $60 \mathrm{~km}$ para el evento del 18 de junio basado en la distribución epicentral de réplicas. Los resultados de Singh et al. (1985) sugieren rupturas independientes que corresponderían a deslizamientos adyacentes en la zona de contacto entre las placas de Rivera y de Norteamérica.

El sismo del 19 de septiembre de 1985 también ocurrió en la zona de subducción de Michoacán-Colima-Jalisco. Este evento corresponde al sismo que más daños económicos ha causado en el país desde que se tienen registros instrumentales, resultando en más de 10000 muertes (Eissler et al., 1986). El sismo tuvo una magnitud de momento (Mw) de 8.0 y fue seguido por otro sismo fuerte con magnitud Mw 7.6 el 21 de septiembre de 1985 (Eissler et al., 1986; UNAM Seismology Group, 1986). Las áreas de réplicas de estos dos grandes sismos fueron de $170 \mathrm{~km}$ x $50 \mathrm{~km}$ para el evento del 19 de septiembre y de $66 \mathrm{~km}$ x $33 \mathrm{~km}$ para el sismo del 21 de septiembre (UNAM Seismology Group, 1986). Estas áreas no se empalman y son consistentes con la ruptura de zonas adyacentes en el contacto interplaca de Cocos-Norteamérica. Los resultados de un análisis de las formas de onda registradas para estos dos eventos indican que efectivamente las rupturas representan el deslizamiento cosísmico de asperezas independientes en la zona de subducción (Mendoza y Hartzell, 1989; Mendoza, 1993).

Estos sismos principales corresponden a fallamiento inverso en la zona de contacto entre las placas CocosRivera y la placa de Norteamérica. Han habido varios sismos interplaca con magnitud mayor a 7 en la región de Michoacán-Colima-Jalisco en el último siglo (Tabla 1). Estos eventos se muestran en la Figura 1 junto con las áreas de réplicas que se han identificado para los sismos registrados a partir de 1932. Si estas áreas representan las zonas de ruptura, entonces estos eventos sugieren la existencia de una zona somera de acoplamiento sismogénico a lo largo de la zona de subducción en la región de Michoacán-Colima-Jalisco.

El contacto interplaca en la zona de subducción de México se ha estudiado anteriormente por varios investigadores (Suárez et al., 1990; Tichelaar y Ruff, 1993; Pacheco et al., 1993; Pardo y Suárez, 1995). Aparte de examinar la geometría de la placa subducida, estos estudios han identificado la profundidad máxima de acoplamiento sismogénico basado en la ubicación de los sismos de fallamiento inverso. Generalmente, un evento se considera como indicador de deslizamiento sismogénico en el contacto interplaca si 1) el rumbo del plano de falla es aproximadamente paralelo a la trinchera y 2) la orientación del vector de deslizamiento es en dirección del buzamiento de la placa subducida y representa principalmente un movimiento de cabalgamiento (Tichelaar y Ruff, 1993). En un estudio de sismos de magnitud mayor a 6 ocurridos durante el período de 1977 a 1988, Tichelaar y Ruff (1993) determinaron que la profundidad máxima de acoplamiento sismogénico a lo largo de la placa de Cocos es de entre 20 y $30 \mathrm{~km}$. Similarmente, en un análisis de la sismicidad observada entre 1964 y 1990, Pardo y Suárez (1995) propusieron una profundidad máxima de $25 \mathrm{~km}$ para la zona sismogénica de la placa de Cocos con un ancho aproximado de $60 \mathrm{~km}$. Esta profundidad máxima es la mitad del valor promedio de 40-55 km observado en otras zonas de subducción del mundo (Tichelaar y Ruff, 1993; Pacheco et al., 1993). Sin embargo, Currie et al. (2002) mencionan que si la intersección del Moho continental con la placa oceánica provee el límite máximo de la zona sismogénica, entonces el ancho sismogénico en la zona de subducción del margen Mexicano podría extenderse de 100 a $145 \mathrm{~km}$. Este valor sería más consistente con las dimensiones de las áreas de réplicas de sismos mayores ocurridos en los últimos años en la costa de Michoacán (Figura 1). Pardo y Suárez (1995) también examinaron la placa de Rivera y estimaron una profundidad máxima de $40 \mathrm{~km}$ para el acoplamiento sismogénico, sugiriendo un ancho de $75 \mathrm{~km}$ tomando un ángulo de buzamiento de $32^{\circ}$ para la placa de Rivera en la región de Colima-Jalisco.

El ancho de la zona sismogénica controla el tamaño de los sismos mayores (Suárez y Sánchez, 1996) y tiene implicaciones importantes para la definición del potencial sísmico, la estimación del movimiento del suelo esperado, y la generación de tsunamis. Los trabajos que se han realizado hasta la fecha han aportado conocimientos importantes sobre la naturaleza del contacto sismogénico en México. Sin embargo, existe información sismológica adicional para la región de Michoacán-Colima-Jalisco que no se ha tomado en cuenta. Por ejemplo, se han producido 
Tabla 1. Sismos interplaca con magnitud mayor o igual a 7 ocurridos de 1900 a 2014 en la zona de subducción de Michoacán-Colima-Jalisco.

\begin{tabular}{|c|c|c|c|}
\hline $\begin{array}{c}\text { Fecha } \\
\text { (día/mes/año) }\end{array}$ & $\begin{array}{l}\text { Latitud } \\
\left(^{\circ}\right) \mathrm{N}\end{array}$ & $\begin{array}{l}\text { Longitud } \\
\left(^{\circ}\right) \mathrm{W}\end{array}$ & Magnitud \\
\hline $20 / 01 / 1900$ & 20.00 & $-105.00^{1}$ & $\mathrm{Mw} 7.4^{14}$ \\
\hline $16 / 05 / 1900$ & 20.00 & $-105.00^{1}$ & Mw $7.0^{14}$ \\
\hline 07/06/1911 & 17.50 & $-102.50^{2}$ & $\mathrm{Mw} 7.6^{14}$ \\
\hline 03/06/1932 & 19.57 & $-104.42^{3}$ & Ms $8.2^{4}$ \\
\hline $18 / 06 / 1932$ & 19.50 & $-103.50^{4}$ & Ms $7.6^{4}$ \\
\hline $15 / 04 / 1941$ & 18.85 & $-102.94^{5}$ & Ms $7.7^{5}$ \\
\hline $22 / 02 / 1943$ & 17.62 & $-101.15^{6}$ & $\mathrm{Mw} 7.4^{14}$ \\
\hline $30 / 01 / 1973$ & 18.39 & $-103.21^{7}$ & $\mathrm{Mw} 7.6^{7}$ \\
\hline $14 / 03 / 1979$ & 17.46 & $-101.45^{8}$ & Mw $7.4^{15}$ \\
\hline $25 / 10 / 1981$ & 17.75 & $-102.25^{9}$ & $\mathrm{Mw} 7.2^{16}$ \\
\hline $19 / 09 / 1985$ & 18.14 & $-102.71^{10}$ & Mw $8.1^{17}$ \\
\hline $21 / 09 / 1985$ & 17.62 & $-101.82^{10}$ & Mw $7.6^{17}$ \\
\hline 09/10/1995 & 18.79 & $-104.47^{11}$ & Mw $7.9^{18}$ \\
\hline $22 / 01 / 2003$ & 18.71 & $-104.13^{12}$ & $\mathrm{Mw} 7.5^{12}$ \\
\hline $18 / 04 / 2014$ & 17.38 & $-101.06^{13}$ & Mw $7.2^{19}$ \\
\hline
\end{tabular}

Los superíndices indican las referencias del epicentro y de la magnitud: 1 Duda, 1965; 2 Gutenberg y Richter, 1954; 3 Eissler y McNally, 1984; 4 Singh et al., 1985; 5 Kelleher et al., 1973; 6 Singh et al., 1981; 7 Reyes et al., 1979; 8 Gettrust et al., 1981; 9 Havskov et al., 1983; 10 UNAM Seismology Group, 1986; 11 Courboulex et al., 1997; 12 Yagi et al., 2004; 13 UNAM Seismology Group, 2015; 14 Anderson et al., 1989; 15 Mendoza, 1995; 16 Mendoza, 1993; 17 Eissler et al., 1986; 18 Mendoza y Hartzell, 1999; 19 CMT, 2013. catálogos sísmicos de alta calidad en los últimos años, incluyendo las relocalizaciones detalladas de Engdahl et al. (1998) para sismos registrados a distancias telesísmicas y la determinación de hipocentros para sismos locales registrados por la red temporal MARS (Mapping the Rivera Subduction Zone) que operó en los estados de Jalisco, Colima y Michoacán. Además, existe una gran cantidad de mecanismos focales para sismos ocurridos en años recientes en la región. El objetivo de este trabajo es examinar las localizaciones de los eventos y los mecanismos focales disponibles hasta la fecha para examinar a detalle el ancho de la zona de acoplamiento sismogénico en la zona de subducción de Michoacán-Colima-Jalisco. Se aplica el mismo procedimiento utilizado anteriormente por otros autores (Suárez y Comte, 1993; Tichelaar y Ruff, 1993; Pardo y Suárez, 1995) donde se examinan las ubicaciones de los sismos de fallamiento inverso y normal para identificar el punto de transición en el plano de contacto interplaca.

\section{Hipocentros y Mecanismos}

Se examinaron los hipocentros relocalizados por Pardo (1993) y utilizados después por Pardo y Suárez (1995) para estudiar la geometría de las placas de Cocos y Rivera. Estos eventos se relocalizaron con el método de Joint Hypocenter Determination de Dewey (1971) y tienen errores hipocentrales menores a $10 \mathrm{~km}$ (Pardo, 1993). Se incluyeron

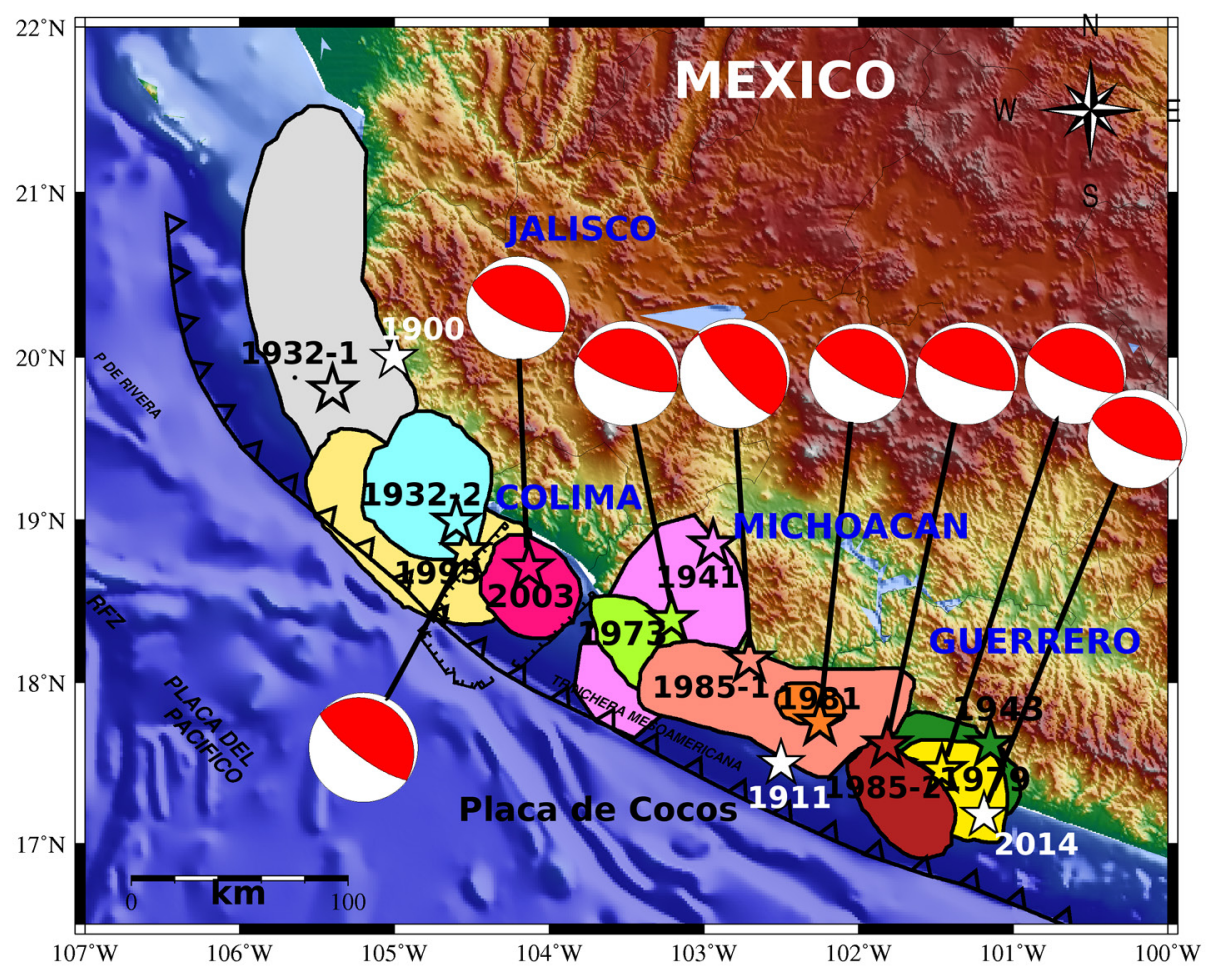

Figura 1. Sismos interplaca mayores o igual a magnitud 7 (estrellas) ocurridos de 1900 a 2014 en la zona de subducción de Michoacán-Colima-Jalisco. Los óvalos indican las áreas de réplicas tomadas de Ramírez-Herrera et al. (2010) y de Kelleher et al. (1973) para sismos ocurridos de 1932 a 2003. Además, se muestran los mecanismos focales para eventos ocurridos a partir de 1973 tomados de Santoyo et al. (2006) y de CMT (2013). 
los sismos con magnitud de ondas de cuerpo $(\mathrm{Mb})$ mayor ó igual a 4.5 ocurridos en el período de 1964 a 1990 . Se consideraron además los eventos mayor o igual a Mb 4.3 del boletín EHB distribuido por el Centro Sismológico Internacional (ISC, 2009) para el período 1964-2008. Estos eventos están relocalizados con la técnica de Engdahl et al. (1998) que utiliza los tiempos de arribo de PKiKP, PKPdf y las fases de profundidad pP, pwP, y sP para restringir los hipocentros. El boletín EHB presenta hasta tres valores de profundidad para cada evento: profundidades fijas, profundidades libres y profundidades determinadas con los tiempos de arribo de la fase pP. Para tener la profundidad más precisa se tomó el valor determinado con la fase $\mathrm{pP}$. Para aquellos eventos donde no se incluye este valor se tomó la profundidad libre. No se consideraron eventos con profundidades fijas. Los eventos del boletín EHB se muestran en la Figura 2 junto con los epicentros de Pardo (1993). Para eventos duplicados durante el período 19641990 se dio preferencia a las localizaciones de Pardo (1993).

También se examinó la microsismicidad local registrada entre enero 2006 a mayo 2007 por la red temporal MARS y localizada por Martínez-López (2011). Este catálogo incluye 110 eventos con magnitud local $\left(\mathrm{M}_{\mathrm{L}}\right)$ entre 1.8 y 5.4 con errores hipocentrales menores a $10 \mathrm{~km}$. Estos eventos se muestran en la Figura 3. Se dio preferencia a estas localizaciones en caso de haber eventos duplicados con los del boletín EHB.

Las figuras 2 y 3 además muestran los mecanismos focales que se consideraron en este trabajo. Estos incluyen los mecanismos utilizados por Pardo y Suárez (1995) para examinar la geometría de la zona de subducción determinados mediante la modelación de las formas de onda de P, SV y SH (Tabla 2). También se incluyen los mecanismos focales determinados por el Global CMT Project (CMT, 2013) para sismos de magnitud Mw mayor a 4.9 ocurridos en el período de 1979 a 2008 (Tabla 3) y las soluciones del Servicio Sismológico Nacional (SSN, 2013) para sismos de magnitud mayor a Mw 4.3 ocurridos dentro del período 2000 - 2001 (Tabla 2). El SSN (2013) determinó estos mecanismos a partir de la inversión de la forma de onda de sismogramas regionales. Se examinaron además los mecanismos focales que obtuvo Martínez-López (2011) con el programa FOCMEC de Snoke et al. (1984) para sismos registrados por la red $M A R S$ entre enero 2006 y mayo 2007 (Tabla 4). Este programa utiliza la polaridad de la onda $\mathrm{P}$ para realizar una búsqueda sistemática de la esfera focal, proporcionando todas las posibles soluciones consistentes con las observaciones. Para los mecanismos duplicados se adoptó el siguiente orden de prioridad: Pardo y Suárez (1995), CMT (2013), SSN (2013) y Martínez-López (2011).

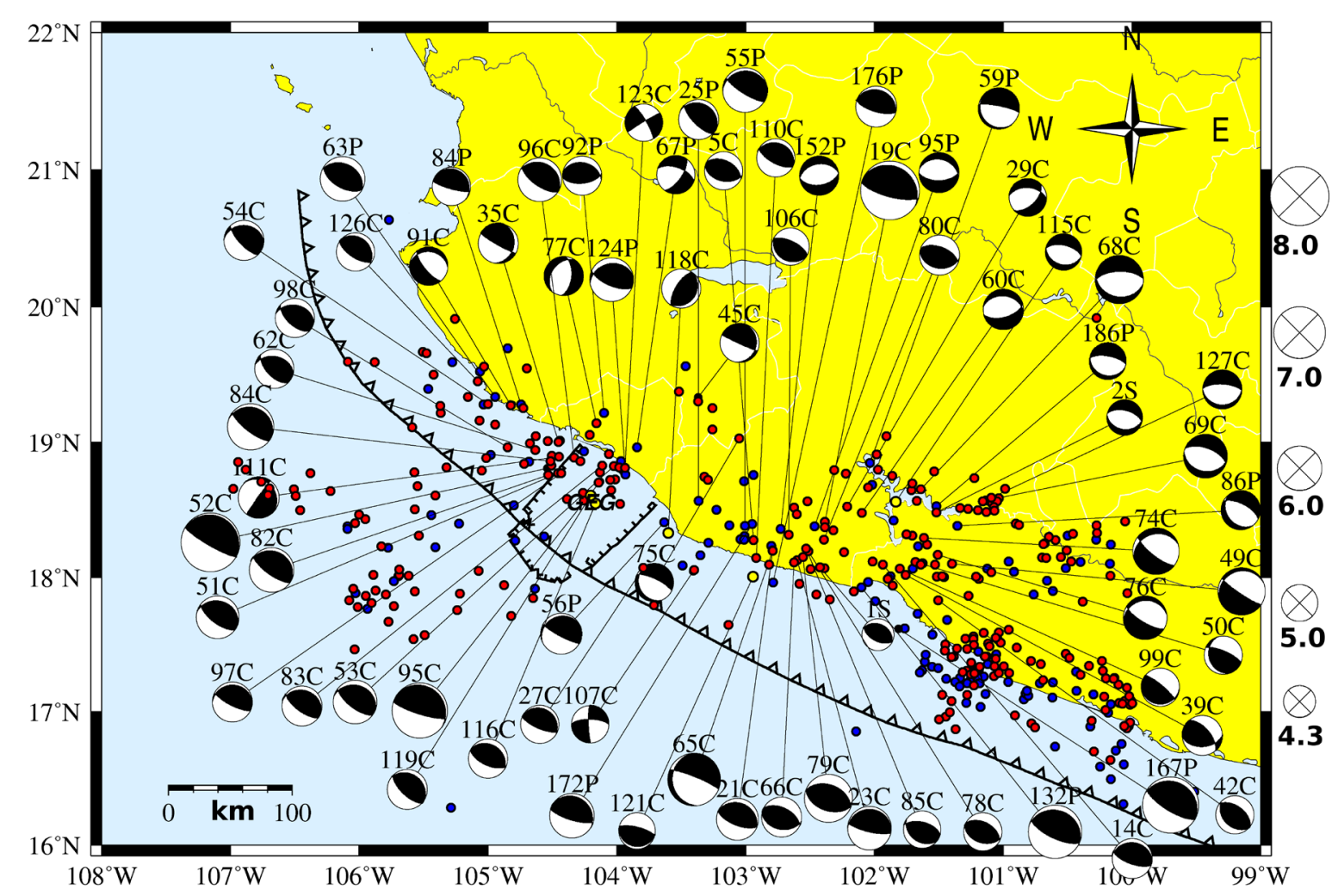

Figura 2. Distribución de la sismicidad entre 1964-2008 relocalizada por Pardo (1993, círculos azules) y obtenida del ISC (2009, círculos rojos). Los mecanismos obtenidos de CMT (2013) entre 1979-2008 se etiquetan con la letra C. Los mecanismos de Pardo y Suárez (1995) están etiquetados con la letra P. Los mecanismos del Servicio Sismológico Nacional (SSN, 2013) en el período de 2000-2001 están etiquetados con la letra S. El tamaño del mecanismo focal representa la magnitud del sismo en base a la escala que se muestra a la derecha. GEG = Graben el Gordo. 


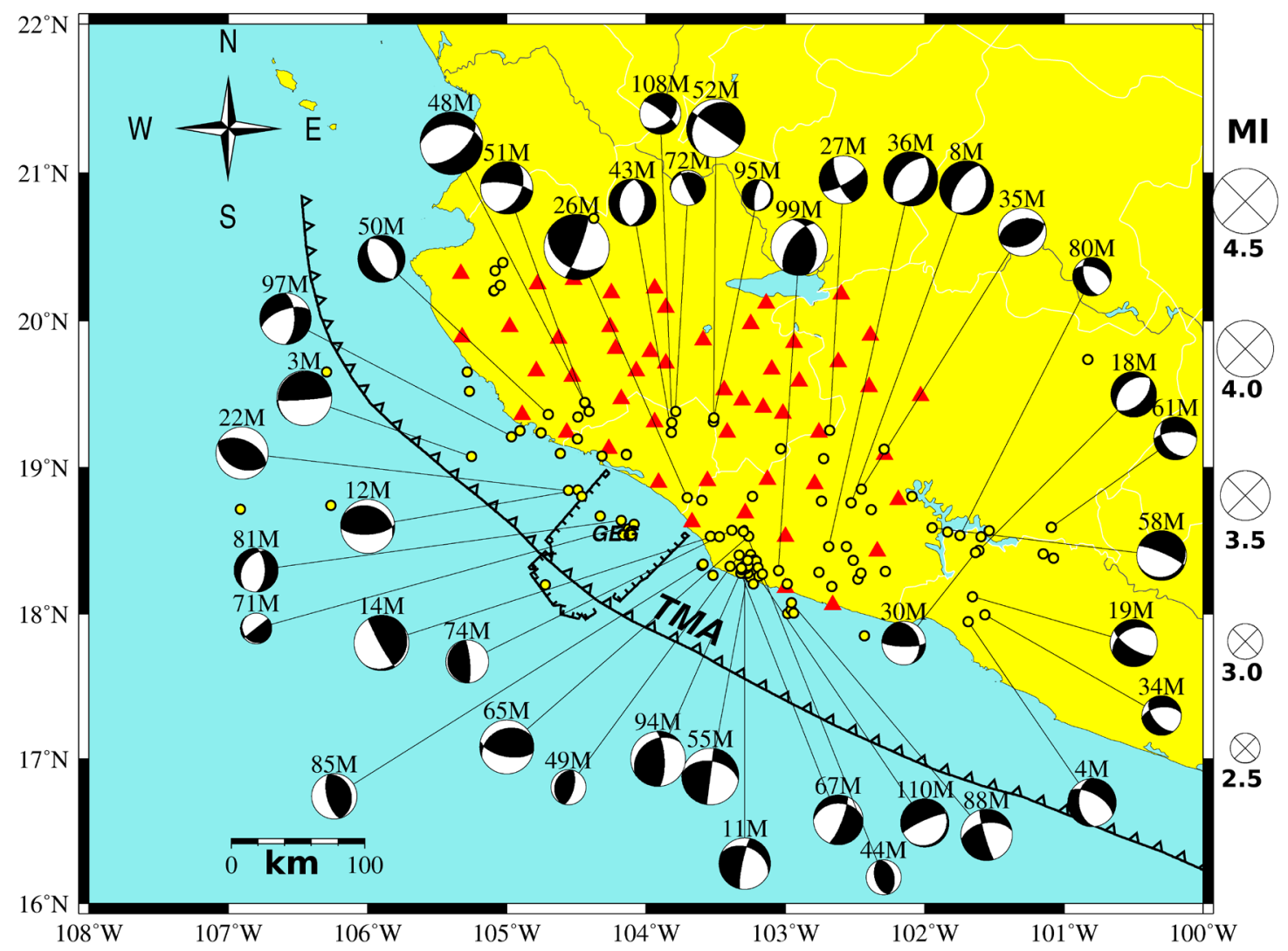

Figura 3. Distribución de la sismicidad (círculos amarillos) y mecanismos focales determinados por Martínez-López (2011) utilizando datos registrados entre enero de 2006 a mayo de 2007 por las estaciones regionales (triángulos rojos) de la red temporal MARS. Los mecanismos están etiquetados con la letra M. GEG $=$ Graben el Gordo y TMA = Trinchera Meso Americana.

Considerando la importancia de identificar con confiabilidad la profundidad máxima del contacto sismogénico en la zona de subducción, se asignaron las profundidades más precisas disponibles para todos los mecanismos. Para los mecanismos de Pardo y Suárez (1995) generalmente se asignaron las profundidades obtenidas por ellos mismos mediante la modelación de las formas de onda. Para los mecanismos CMT (2013) y del SSN (2013) se adoptaron las profundidades del boletín EHB determinadas utilizando la fase pP. Para los mecanismos CMT (2013) que no cuentan con este valor, se tomó la profundidad del centroide del tensor de momento. Además, se examinaron los estudios anteriores de sismos mayores a magnitud 7 para identificar la profundidad conocida de estos eventos.

\section{Análisis y Resultados}

Para visualizar la distribución de la sismicidad en profundidad, se realizaron nueve perfiles perpendiculares a la trinchera Mesoamericana en la región de estudio (Figura 4). Estos perfiles se extienden lateralmente a lo largo de los segmentos de Jalisco y de Michoacán previamente definidos por Pardo y Suárez (1995). El segmento de Michoacán corresponde a la subducción de la porción noroeste de la placa de Cocos desde el Graben el Gordo hasta la intersección con la Zona de Fractura de Orozco. El segmento de Jalisco corresponde a la subducción de la placa de Rivera y se extiende desde el Graben el Gordo hacia el noroeste hasta una longitud de $-106^{\circ}$ (Pardo y Suárez, 1995). Hacia el norte, asumimos que el segmento se extiende hasta una latitud de $21.5^{\circ}$ basado en la ubicación de las réplicas del sismo de 1932 (ver Figura 1).

La dirección de los perfiles es generalmente SO-NE, con una longitud aproximada de $280 \mathrm{~km}$ y un ancho de 50 $\mathrm{km}$ cada uno. En los perfiles se grafican los hipocentros de Pardo (1993), los eventos del boletín EHB, los mecanismos focales de Pardo y Suárez (1995), la microsismicidad local y mecanismos de Martínez-López (2011), y los mecanismos focales del CMT (2013) y del SSN (2013). El límite entre las placas de Rivera y de Cocos a lo largo de la trinchera se ha discutido por diferentes autores sin haber una clara definición hasta el momento (Larson, 1972; Bandy et al., 1995; 1998; Serrato-Díaz et al., 2004; Yang et al., 2009; Peláez-Gaviria et al., 2013). Bandy et al. $(1995 ; 1998)$ proponen que el Graben el Gordo podría indicar la transición entre las placas de Rivera y de Cocos. Ellos mencionan que el límite a profundidad podría estar al este del Centro y Norte del rift de Colima, lo cual concuerda con la variación de la geometría de la placa propuesta por Pardo y Suárez 
Tabla 2. Mecanismos focales de Pardo y Suárez (1995) (P) y del Servicio Sismológico Nacional (S) analizados en este estudio.

\begin{tabular}{|c|c|c|c|c|c|c|c|c|}
\hline \multirow[b]{2}{*}{ Evento } & \multirow{2}{*}{$\begin{array}{c}\text { Fecha } \\
\text { (día/mes/año) }\end{array}$} & \multirow[b]{2}{*}{$\begin{array}{l}\text { Latitud } \\
\qquad\left(^{\circ}\right)\end{array}$} & \multirow{2}{*}{$\begin{array}{l}\text { Hipocentro* } \\
\text { Longitud } \\
\left({ }^{\circ}\right)\end{array}$} & \multirow[b]{2}{*}{$\begin{array}{c}\mathrm{H} \\
(\mathrm{km})\end{array}$} & \multicolumn{3}{|c|}{ Mecanismo** } & \multirow[b]{2}{*}{ Magnitud } \\
\hline & & & & & $\begin{array}{l}\mathrm{R}_{\mathrm{m}} \\
\left(^{\circ}\right)\end{array}$ & $\begin{array}{l}\mathrm{B}_{\mathrm{z}} \\
\left({ }^{\circ}\right)\end{array}$ & $\begin{array}{l}A_{d} \\
\left({ }^{\circ}\right)\end{array}$ & \\
\hline $25 \mathrm{P}$ & $26 / 04 / 1968$ & $18.568^{1}$ & -103.567 & $16.0^{1}$ & 297 & 21 & 73 & $5.5 \mathrm{Mb}$ \\
\hline $55 \mathrm{P}$ & $30 / 01 / 1973$ & $18.383^{1}$ & -103.004 & $16.5^{3}$ & 266 & 17 & 55 & $7.7 \mathrm{Mw}$ \\
\hline $56 \mathrm{P}$ & $10 / 02 / 1973$ & $18.410^{1}$ & -103.631 & $11.0^{1}$ & 246 & 16 & 39 & $5.6 \mathrm{Mb}$ \\
\hline $59 \mathrm{P}$ & 03/07/1973 & $18.848^{1}$ & -102.034 & $94.9^{1}$ & 281 & 80 & -98 & $5.6 \mathrm{Mb}$ \\
\hline $63 \mathrm{P}$ & $18 / 10 / 1973$ & $19.337^{1}$ & -104.945 & $13.5^{1}$ & 296 & 33 & 86 & $6.0 \mathrm{Mb}$ \\
\hline $67 \mathrm{P}$ & $26 / 01 / 1974$ & $18.966^{1}$ & -103.843 & $50.8^{1}$ & 35 & 65 & -145 & $5.2 \mathrm{Mb}$ \\
\hline $84 \mathrm{P}$ & 01/07/1976 & $19.280^{1}$ & -104.744 & $13.3^{1}$ & 311 & 12 & 115 & $5.2 \mathrm{Mb}$ \\
\hline $86 \mathrm{P}$ & 05/09/1976 & $18.384^{1}$ & -101.356 & $63.3^{1}$ & 297 & 64 & -102 & $5.3 \mathrm{Mb}$ \\
\hline $92 \mathrm{P}$ & $25 / 04 / 1978$ & $19.219^{1}$ & -104.100 & $38.8^{1}$ & 282 & 34 & 105 & $5.3 \mathrm{Mb}$ \\
\hline $95 \mathrm{P}$ & 29/09/1978 & $18.380^{1}$ & -102.464 & $62.8^{1}$ & 281 & 33 & -80 & $5.4 \mathrm{Mb}$ \\
\hline $124 \mathrm{P}$ & 09/03/1981 & $18.762^{1}$ & -103.935 & $22.2^{1}$ & 260 & 28 & 60 & $5.9 \mathrm{Mb}$ \\
\hline $132 \mathrm{P}$ & $25 / 10 / 1981$ & $17.928^{1}$ & -102.101 & $20.0^{4}$ & 287 & 20 & 82 & $7.2 \mathrm{Mw}$ \\
\hline $152 \mathrm{P}$ & $08 / 12 / 1983$ & $18.359^{1}$ & -102.729 & $53.7^{1}$ & 262 & 40 & -90 & $5.3 \mathrm{Mw}$ \\
\hline $167 \mathrm{P}$ & $21 / 09 / 1985$ & $17.621^{1}$ & -101.762 & $22.0^{5}$ & 296 & 17 & 85 & $7.8 \mathrm{Mw}$ \\
\hline $172 \mathrm{P}$ & $30 / 04 / 1985$ & $18.371^{1}$ & -103.000 & $22.7^{1}$ & 303 & 16 & 105 & $5.6 \mathrm{Mb}$ \\
\hline $176 \mathrm{P}$ & 05/05/1986 & $17.963^{1}$ & -102.788 & $16.6^{1}$ & 288 & 21 & 91 & $6.1 \mathrm{Mb}$ \\
\hline $186 \mathrm{P}$ & $26 / 07 / 1987$ & $18.530^{1}$ & -101.520 & $74.1^{1}$ & 280 & 63 & -93 & $5.1 \mathrm{Mb}$ \\
\hline $1 \mathrm{~S}$ & $10 / 08 / 2000$ & $18.076^{2}$ & -102.424 & $27.1^{2}$ & 294 & 35 & 87 & $4.3 \mathrm{Mw}$ \\
\hline $2 \mathrm{~S}$ & $11 / 02 / 2001$ & $18.484^{2}$ & -101.518 & $47.4^{2}$ & 284 & 57 & -87 & $4.8 \mathrm{Mw}$ \\
\hline
\end{tabular}

* Los superíndices indican las referencias del epicentro y de la profundidad (H): 1 Pardo (1993); 2 ISC (2009); 3 Santoyo et al. (2006); 4 Priestley y Masters (1986); 5 Eissler et al. (1986).

** $\mathrm{Rm}=$ Rumbo, $\mathrm{Bz}=$ Buzamiento, $\mathrm{Ad}=$ Ángulo de deslizamiento.

(1995). DeMets y Wilson (1997) proponen que la frontera es una zona difusa de cizallamiento lateral izquierdo con orientación NNE-SSO. En este trabajo se asume que el límite entre las placas Cocos y Rivera se encuentra en el Graben el Gordo entre los perfiles E-E' y F-F' en la Figura 4.

\subsection{Segmento de Michoacán}

En la Figura 5 se muestran los perfiles que se analizaron para examinar la zona de acoplamiento sismogénico de la placa de Cocos en el segmento de Michoacán. Los perfiles muestran la proyección lateral de los mecanismos focales: los rojos indican fallamiento inverso consistente con deslizamiento en el contacto interplaca y los azules indican fallamiento normal. Todos los demás mecanismos se muestran en color gris e incluyen mecanismos inversos inconsistentes con deslizamiento en el contacto interplaca $\mathrm{y}$ eventos de fallamiento transcurrente.

En el perfil A-A' (Figura 5a) se observa sismicidad hasta aproximadamente $80 \mathrm{~km}$ de profundidad a $220 \mathrm{~km}$ de distancia de la trinchera. El mecanismo inverso más profundo consistente con el contacto sismogénico (evento
50C) está a una profundidad de $38 \mathrm{~km}$ y se encuentra a 130 $\mathrm{km}$ de la trinchera aproximadamente. El evento inverso $30 \mathrm{M}$ a $75 \mathrm{~km}$ de profundidad se encuentra dentro de la placa subducente. Ohta et al. (2011) mencionan que este tipo de sismos inversos son generados por la reactivación de zonas débiles preexistentes hidratadas. También se observan eventos de fallamiento normal a profundidades mayores a $60 \mathrm{~km}$ que se encuentran a más de $140 \mathrm{~km}$ de la trinchera. En el perfil B-B' (Figura 5b) se observan dos eventos de fallamiento inverso (eventos 132P y 14C) con una profundidad máxima de $24 \mathrm{~km}$. En el perfil C-C' (Figura 5c) se observan varios eventos de fallamiento inverso consistentes con deslizamiento en el contacto interplaca hasta una profundidad de $40 \mathrm{~km}$ (evento $78 \mathrm{C}$ ). El evento $65 \mathrm{C}$ de fallamiento normal a $35 \mathrm{~km}$ de profundidad corresponde al sismo Mw 7.1 del 11 de enero de 1997 que ocurrió dentro de la placa de Cocos (Santoyo et al., 2005) debajo de la zona de ruptura del sismo Mw 8.0 del 19 de septiembre de 1985 (evento 19C). El evento inverso 35M a $80 \mathrm{~km}$ de profundidad es parecido al evento $30 \mathrm{M}$ del perfil A-A' y está dentro de la placa subducente. En el perfil D-D' (Figura 5d) varios eventos someros muestran fallamiento 
Tabla 3. Mecanismos focales del CMT analizados en este estudio.

\begin{tabular}{|c|c|c|c|c|c|c|c|c|}
\hline \multirow[b]{2}{*}{ Evento } & \multirow[b]{2}{*}{$\begin{array}{c}\text { Fecha } \\
\text { (día/mes/año) }\end{array}$} & \multicolumn{3}{|c|}{ Hipocentro* } & \multicolumn{3}{|c|}{ Mecanismo ** } & \multirow{2}{*}{$\begin{array}{l}\text { Magnitud de } \\
\text { Momento } \\
(\mathrm{Mw})\end{array}$} \\
\hline & & $\begin{array}{l}\text { Latitud } \\
\qquad\left(^{\circ}\right)\end{array}$ & $\begin{array}{c}\text { Longitud } \\
\quad\left({ }^{\circ}\right)\end{array}$ & $\begin{array}{c}\mathrm{H} \\
(\mathrm{km})\end{array}$ & $\begin{array}{l}\mathrm{R}_{\mathrm{m}} \\
\left({ }^{\circ}\right)\end{array}$ & $\begin{array}{l}\mathrm{B}_{\mathrm{z}} \\
\left({ }^{\circ}\right)\end{array}$ & $\begin{array}{l}\mathrm{A}_{\mathrm{d}} \\
\left({ }^{\circ}\right)\end{array}$ & \\
\hline $5 \mathrm{C}$ & $06 / 01 / 1979$ & $18.278^{1}$ & -102.940 & $28.8^{1}$ & 103 & 44 & 80 & 5.2 \\
\hline $14 \mathrm{C}$ & $26 / 07 / 1981$ & $18.097^{1}$ & -101.936 & $18.3^{1}$ & 276 & 23 & 77 & 5.5 \\
\hline $19 \mathrm{C}$ & $19 / 09 / 1985$ & $18.414^{1}$ & -102.385 & $17.0^{3}$ & 301 & 18 & 105 & 8.0 \\
\hline $21 \mathrm{C}$ & $25 / 09 / 1985$ & $18.201^{1}$ & -102.791 & $22.9^{4}$ & 293 & 24 & 91 & 5.7 \\
\hline $23 \mathrm{C}$ & $29 / 10 / 1985$ & $18.128^{1}$ & -102.599 & $31.2^{1}$ & 270 & 15 & 77 & 5.9 \\
\hline $27 \mathrm{C}$ & $27 / 09 / 1987$ & $18.071^{1}$ & -103.794 & $15.0^{1}$ & 300 & 18 & 96 & 5.2 \\
\hline $29 \mathrm{C}$ & $14 / 09 / 1988$ & $18.526^{1}$ & -102.211 & $78.9^{1}$ & 274 & 47 & -53 & 5.1 \\
\hline $35 \mathrm{C}$ & 08/12/1989 & $19.005^{1}$ & -104.454 & $15.0^{4}$ & 13 & 22 & 162 & 5.5 \\
\hline $39 \mathrm{C}$ & $14 / 01 / 1991$ & $18.021^{1}$ & -101.757 & $36.7^{1}$ & 87 & 44 & 47 & 5.5 \\
\hline $42 \mathrm{C}$ & $12 / 02 / 1992$ & $17.832^{1}$ & -101.504 & $35.5^{1}$ & 134 & 38 & 96 & 5.2 \\
\hline $45 \mathrm{C}$ & 29/09/1992 & $19.302^{1}$ & -103.368 & $93.8^{1}$ & 12 & 16 & 167 & 5.3 \\
\hline $49 \mathrm{C}$ & $10 / 12 / 1994$ & $18.103^{1}$ & -101.397 & $59.6^{1}$ & 247 & 15 & -146 & 6.4 \\
\hline $50 \mathrm{C}$ & $27 / 04 / 1995$ & $18.116^{1}$ & -101.681 & $38.3^{1}$ & 162 & 25 & 136 & 5.2 \\
\hline $51 \mathrm{C}$ & $06 / 10 / 1995$ & $18.774^{1}$ & -104.458 & $25.2^{1}$ & 285 & 25 & 70 & 5.8 \\
\hline $52 \mathrm{C}$ & 09/10/1995 & $19.056^{1}$ & -104.210 & $17.0^{5}$ & 302 & 9 & 92 & 8.0 \\
\hline $53 \mathrm{C}$ & $12 / 10 / 1995$ & $18.826^{1}$ & -104.023 & $20.1^{1}$ & 286 & 23 & 70 & 5.9 \\
\hline $54 \mathrm{C}$ & $18 / 10 / 1995$ & $19.285^{1}$ & -105.001 & $16.8^{4}$ & 273 & 25 & 49 & 5.4 \\
\hline $60 \mathrm{C}$ & $25 / 01 / 1996$ & $18.481^{1}$ & -102.098 & $73.2^{2}$ & 276 & 34 & -72 & 5.5 \\
\hline $62 \mathrm{C}$ & 09/05/1996 & $18.944^{1}$ & -104.633 & $15.0^{7}$ & 281 & 37 & 60 & 5.3 \\
\hline $65 \mathrm{C}$ & $11 / 01 / 1997$ & $18.198^{1}$ & -102.790 & $35.0^{6}$ & 175 & 18 & -28 & 7.1 \\
\hline $66 \mathrm{C}$ & $16 / 01 / 1997$ & $18.113^{1}$ & -102.623 & $28.7^{2}$ & 102 & 38 & 79 & 5.5 \\
\hline $68 \mathrm{C}$ & $22 / 05 / 1997$ & $18.653^{1}$ & -101.642 & $91.3^{2}$ & 102 & 28 & -78 & 6.5 \\
\hline $69 \mathrm{C}$ & $20 / 04 / 1998$ & $18.571^{1}$ & -101.134 & $62.0^{2}$ & 101 & 30 & -98 & 5.9 \\
\hline $74 \mathrm{C}$ & $21 / 06 / 1999$ & $18.293^{1}$ & -101.617 & $67.7^{2}$ & 254 & 27 & -141 & 6.3 \\
\hline $75 \mathrm{C}$ & $15 / 08 / 1999$ & $19.029^{1}$ & -103.049 & $83.2^{2}$ & 156 & 21 & -51 & 5.2 \\
\hline $76 \mathrm{C}$ & $29 / 12 / 1999$ & $18.170^{1}$ & -101.509 & $67.2^{2}$ & 260 & 22 & -129 & 5.9 \\
\hline $77 \mathrm{C}$ & $07 / 03 / 2000$ & $19.144^{1}$ & -104.160 & $15.0^{4}$ & 215 & 37 & -66 & 5.3 \\
\hline $78 \mathrm{C}$ & $11 / 04 / 2000$ & $18.196^{1}$ & -102.513 & $40.1^{1}$ & 109 & 42 & 84 & 5.2 \\
\hline $79 \mathrm{C}$ & $09 / 08 / 2000$ & $18.151^{1}$ & -102.556 & $20.4^{1}$ & 291 & 43 & 91 & 6.5 \\
\hline $80 \mathrm{C}$ & $01 / 12 / 2000$ & $18.280^{1}$ & -102.394 & $23.1^{1}$ & 99 & 34 & 80 & 5.4 \\
\hline $82 \mathrm{C}$ & $29 / 04 / 2001$ & $18.750^{1}$ & -104.534 & $27.5^{1}$ & 292 & 18 & 77 & 6.1 \\
\hline $83 \mathrm{C}$ & $08 / 05 / 2001$ & $18.831^{1}$ & -104.114 & $22.0^{1}$ & 284 & 23 & 69 & 5.5 \\
\hline $84 \mathrm{C}$ & $20 / 05 / 2001$ & $18.862^{1}$ & -104.514 & $31.8^{1}$ & 290 & 17 & 73 & 6.3 \\
\hline $85 \mathrm{C}$ & $16 / 06 / 2001$ & $18.216^{1}$ & -102.532 & $38.3^{1}$ & 126 & 35 & 108 & 5.1 \\
\hline $91 \mathrm{C}$ & $05 / 11 / 2002$ & $19.274^{1}$ & -104.825 & $15.0^{4}$ & 277 & 26 & -131 & 5.2 \\
\hline $95 \mathrm{C}$ & $22 / 01 / 2003$ & $18.914^{1}$ & -104.062 & $20.0^{7}$ & 308 & 12 & 110 & 7.5 \\
\hline $96 \mathrm{C}$ & $22 / 01 / 2003$ & $18.889^{1}$ & -104.333 & $42.3^{1}$ & 299 & 23 & 85 & 5.9 \\
\hline $97 \mathrm{C}$ & $22 / 01 / 2003$ & $18.773^{1}$ & -104.434 & $21.6^{1}$ & 271 & 16 & 65 & 5.4 \\
\hline $98 \mathrm{C}$ & $17 / 02 / 2003$ & $18.963^{1}$ & -104.848 & $18.1^{4}$ & 282 & 34 & 64 & 5.3 \\
\hline $99 \mathrm{C}$ & $27 / 03 / 2003$ & $18.065^{1}$ & -101.805 & $33.4^{1}$ & 94 & 13 & 55 & 5.2 \\
\hline $106 \mathrm{C}$ & $06 / 02 / 2004$ & $18.319^{1}$ & -102.666 & $27.8^{1}$ & 120 & 25 & 94 & 5.1 \\
\hline $107 \mathrm{C}$ & $13 / 03 / 2004$ & $18.057^{1}$ & -103.402 & $31.9^{1}$ & 271 & 69 & -172 & 5.1 \\
\hline $110 \mathrm{C}$ & $21 / 05 / 2004$ & $18.145^{1}$ & -102.918 & $25.3^{1}$ & 287 & 24 & 79 & 5.2 \\
\hline $111 \mathrm{C}$ & $28 / 02 / 2005$ & $18.894^{1}$ & -104.450 & $24.6^{1}$ & 315 & 19 & 10 & 5.6 \\
\hline $115 \mathrm{C}$ & $20 / 03 / 2006$ & $18.562^{2}$ & -101.836 & $74.2^{2}$ & 105 & 31 & -88 & 4.9 \\
\hline $116 \mathrm{C}$ & $31 / 07 / 2006$ & $18.548^{2}$ & -104.166 & $10.0^{2}$ & 302 & 28 & 96 & 5.3 \\
\hline $118 \mathrm{C}$ & $13 / 08 / 2006$ & $18.332^{2}$ & -103.600 & $15.8^{2}$ & 38 & 23 & 96 & 5.3 \\
\hline $119 \mathrm{C}$ & $19 / 11 / 2006$ & $18.608^{2}$ & -104.204 & $6.0^{2}$ & 305 & 23 & 82 & 5.5 \\
\hline $121 \mathrm{C}$ & $13 / 04 / 2007$ & $18.006^{2}$ & -102.943 & $6.9^{2}$ & 98 & 17 & 84 & 5.0 \\
\hline $123 \mathrm{C}$ & $31 / 05 / 2007$ & $18.812^{1}$ & -103.936 & $22.2^{4}$ & 330 & 79 & -176 & 5.1 \\
\hline $126 \mathrm{C}$ & $06 / 09 / 2007$ & $19.452^{1}$ & -105.079 & $32.8^{1}$ & 310 & 25 & 96 & 5.2 \\
\hline $127 \mathrm{C}$ & $29 / 04 / 2008$ & $18.591^{1}$ & -101.095 & $65.4^{1}$ & 275 & 62 & -86 & 5.3 \\
\hline
\end{tabular}

* Los superíndices indican las referencias del epicentro y de la profundidad (H): 1 ISC (2009); 2 Martínez-López (2011); 3 Eissler et al. (1986); 4 Global CMT Project; 5 Courboulex et al. (1997); 6 Santoyo et al. (2005); 7 Yagi et al. (2004).

$* * \mathrm{Rm}=$ Rumbo, $\mathrm{Bz}=$ Buzamiento, $\mathrm{Ad}=$ Ángulo de deslizamiento. 
Tabla 4. Hipocentros y mecanismos focales de Martínez-López (2011) analizados en este estudio.

\begin{tabular}{|c|c|c|c|c|c|c|c|c|}
\hline \multirow[b]{2}{*}{ Evento } & \multirow{2}{*}{$\begin{array}{c}\text { Fecha } \\
\text { (día/mes/año) }\end{array}$} & \multicolumn{3}{|c|}{ Hipocentro } & \multicolumn{3}{|c|}{ Mecanismo** } & \multirow{2}{*}{$\begin{array}{c}\text { Magnitud } \\
\text { Local } \\
\left(\mathrm{M}_{\mathrm{L}}\right)\end{array}$} \\
\hline & & $\begin{array}{l}\text { Latitud } \\
\qquad\left(^{\circ}\right)\end{array}$ & $\begin{array}{l}\text { Longitud } \\
\qquad\left(^{\circ}\right)\end{array}$ & $\begin{array}{c}\mathrm{H}^{*} \\
(\mathrm{~km})\end{array}$ & $\begin{array}{l}\mathrm{R}_{\mathrm{m}} \\
\left({ }^{\circ}\right)\end{array}$ & $\begin{array}{l}\mathrm{B}_{\mathrm{z}} \\
\left({ }^{\circ}\right)\end{array}$ & $\begin{array}{l}\mathrm{A}_{\mathrm{d}} \\
\left({ }^{\circ}\right)\end{array}$ & \\
\hline $3 \mathrm{M}$ & $17 / 02 / 2006$ & 19.076 & -105.255 & 11.5 & 220 & 7 & 45 & 4.1 \\
\hline $4 \mathrm{M}$ & $17 / 02 / 2006$ & 17.945 & -101.687 & 11.6 & 175 & 45 & -45 & 3.2 \\
\hline $8 \mathrm{M}$ & $17 / 03 / 2006$ & 18.760 & -102.529 & 71.0 & 213 & 60 & -84 & 3.7 \\
\hline $11 \mathrm{M}$ & $26 / 03 / 2006$ & 18.278 & -103.292 & 5.7 & 192 & 82 & -55 & 3.6 \\
\hline $12 \mathrm{M}$ & $29 / 03 / 2006$ & 18.848 & -104.489 & 8.6 & 86 & 65 & 79 & 3.8 \\
\hline $14 \mathrm{M}$ & 03/04/2006 & 18.532 & -103.538 & 18.1 & 158 & 81 & 70 & 3.9 \\
\hline $18 \mathrm{M}$ & $16 / 04 / 2006$ & 18.436 & -101.611 & 32.5 & 225 & 40 & -90 & 3.7 \\
\hline $19 \mathrm{M}$ & $22 / 04 / 2006$ & 18.118 & -101.657 & 12.6 & 124 & 69 & 58 & 3.3 \\
\hline $22 \mathrm{M}$ & $01 / 05 / 2006$ & 18.846 & -104.559 & 5.5 & 115 & 40 & 90 & 3.7 \\
\hline $26 \mathrm{M}$ & $18 / 05 / 2006$ & 18.797 & -103.704 & 45.7 & 122 & 36 & 14 & 4.6 \\
\hline $27 \mathrm{M}$ & $19 / 05 / 2006$ & 19.255 & -102.684 & 4.5 & 61 & 74 & -20 & 3.4 \\
\hline $30 \mathrm{M}$ & $30 / 05 / 2006$ & 18.420 & -101.636 & 74.1 & 91 & 77 & 59 & 3.8 \\
\hline $32 \mathrm{M}$ & 07/06/2006 & 18.531 & -101.593 & 78.4 & 145 & 41 & -40 & 2.8 \\
\hline $34 \mathrm{M}$ & $29 / 06 / 2006$ & 17.993 & -101.569 & 8.2 & 143 & 55 & -45 & 2.8 \\
\hline $35 \mathrm{M}$ & $16 / 07 / 2006$ & 18.855 & -102.456 & 81.5 & 65 & 45 & 83 & 3.4 \\
\hline $36 \mathrm{M}$ & $17 / 07 / 2006$ & 18.461 & -102.689 & 6.8 & 220 & 50 & -83 & 3.8 \\
\hline $43 \mathrm{M}$ & $05 / 08 / 2006$ & 19.240 & -103.818 & 15.3 & 185 & 45 & -90 & 3.3 \\
\hline $44 \mathrm{M}$ & $11 / 08 / 2006$ & 18.576 & -103.300 & 38.0 & 165 & 50 & 90 & 2.5 \\
\hline $48 \mathrm{M}$ & $16 / 09 / 2006$ & 19.440 & -104.438 & 16.2 & 270 & 40 & -58 & 4.4 \\
\hline $49 \mathrm{M}$ & $18 / 09 / 2006$ & 18.415 & -103.347 & 5.4 & 15 & 65 & 90 & 2.5 \\
\hline $50 \mathrm{M}$ & $20 / 09 / 2006$ & 19.363 & -104.704 & 39.4 & 330 & 40 & -90 & 3.3 \\
\hline $51 \mathrm{M}$ & $13 / 10 / 2006$ & 19.445 & -104.440 & 17.8 & 279 & 75 & -48 & 3.7 \\
\hline $52 \mathrm{M}$ & $14 / 10 / 2006$ & 19.315 & -103.516 & 87.2 & 215 & 25 & 0 & 3.7 \\
\hline $55 \mathrm{M}$ & $21 / 10 / 2006$ & 18.280 & -103.270 & 5.0 & 7 & 90 & 45 & 4.0 \\
\hline $58 \mathrm{M}$ & $30 / 10 / 2006$ & 18.592 & -101.948 & 61.3 & 293 & 80 & -80 & 3.5 \\
\hline $61 \mathrm{M}$ & $28 / 11 / 2006$ & 18.594 & -101.091 & 20.3 & 145 & 41 & -41 & 3.0 \\
\hline $65 \mathrm{M}$ & $27 / 12 / 2006$ & 18.533 & -103.266 & 22.3 & 248 & 33 & 62 & 3.8 \\
\hline $67 \mathrm{M}$ & $29 / 12 / 2006$ & 18.279 & -103.320 & 5.4 & 271 & 33 & -24 & 3.7 \\
\hline $71 \mathrm{M}$ & 07/01/2007 & 18.615 & -104.088 & 10.7 & 231 & 90 & 75 & 2.1 \\
\hline $72 \mathrm{M}$ & 08/01/2007 & 19.383 & -103.788 & 18.1 & 263 & 16 & 18 & 2.5 \\
\hline $74 \mathrm{M}$ & $21 / 06 / 2007$ & 18.295 & -101.614 & 47.0 & 254 & 27 & -141 & 6.3 \\
\hline $80 \mathrm{M}$ & $04 / 02 / 2007$ & 18.537 & -101.748 & 35.4 & 162 & 56 & -53 & 2.7 \\
\hline $81 \mathrm{M}$ & $11 / 02 / 2007$ & 18.641 & -104.179 & 6.1 & 211 & 38 & -65 & 3.1 \\
\hline $85 \mathrm{M}$ & $01 / 03 / 2007$ & 18.339 & -103.592 & 16.7 & 165 & 65 & 90 & 3.2 \\
\hline $88 \mathrm{M}$ & 07/03/2007 & 18.075 & -102.956 & 7.6 & 164 & 84 & -40 & 3.6 \\
\hline $94 \mathrm{M}$ & $02 / 04 / 2007$ & 18.302 & -103.314 & 18.0 & 357 & 72 & 64 & 3.8 \\
\hline $95 \mathrm{M}$ & $04 / 04 / 2007$ & 19.340 & -103.515 & 13.8 & 190 & 75 & -90 & 2.2 \\
\hline $97 \mathrm{M}$ & $10 / 04 / 2007$ & 19.212 & -104.966 & 14.1 & 251 & 60 & -35 & 3.6 \\
\hline $99 \mathrm{M}$ & $13 / 04 / 2007$ & 18.298 & -103.048 & 7.7 & 346 & 42 & 51 & 3.6 \\
\hline $108 \mathrm{M}$ & $13 / 05 / 2007$ & 19.307 & -103.816 & 6.7 & 308 & 79 & -49 & 2.9 \\
\hline $110 \mathrm{M}$ & $17 / 05 / 2007$ & 18.258 & -103.190 & 12.8 & 246 & 80 & -85 & 3.4 \\
\hline
\end{tabular}

* $\mathrm{H}=$ Profundidad

** $\mathrm{Rm}=$ Rumbo, $\mathrm{Bz}=$ Buzamiento, $\mathrm{Ad}=$ Ángulo de deslizamiento. 


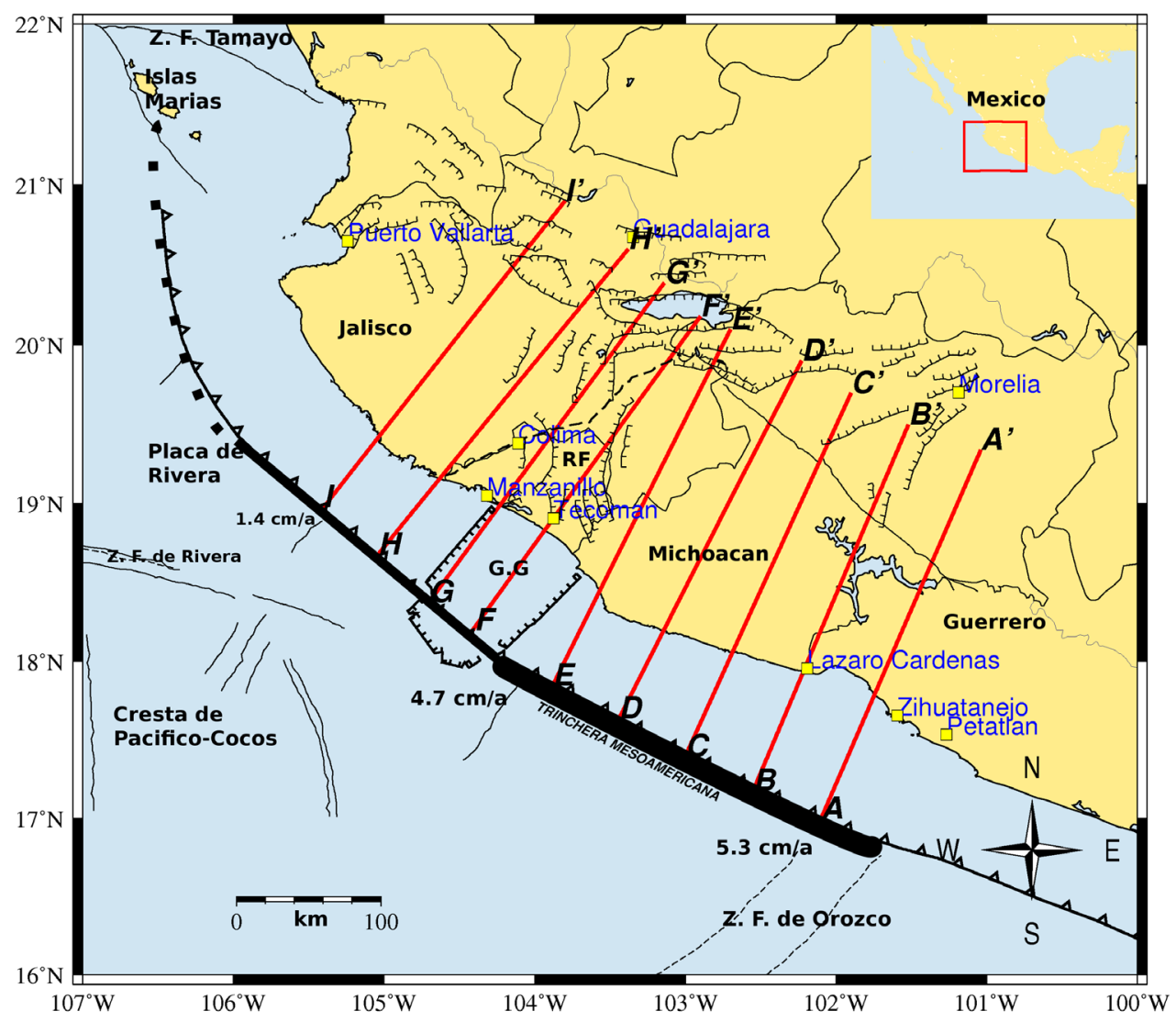

Figura 4. Perfiles perpendiculares a la Trinchera Mesoaméricana en la región de Michoacán-Colima-Jalisco. Las líneas rojas indican la ubicación de los perfiles. La línea negra gruesa indica la longitud del segmento de Michoacán definido por Pardo y Suárez (1995). La línea continua de color negro indica la longitud del segmento de Jalisco (Pardo y Suárez, 1995), inferida hacia el norte (línea punteada) en base a las replicas observadas para el sismo de 1932. G.G. $=$ Graben el Gordo, Z. F. = Zona de Fractura y RF $=$ Rift de Colima.

oblicuo normal e inverso inconsistente con la geometría del contacto interplaca. Los eventos de fallamiento inverso consistente con deslizamiento en la zona de contacto se extienden hasta una profundidad de $28 \mathrm{~km}$ y no se observan mecanismos normales dentro de la placa subducente. En el perfil E-E' (Figura 5e) se observa fallamiento inverso hasta una profundidad de $22 \mathrm{~km}$ y un evento normal a una profundidad de aproximadamente $85 \mathrm{~km}$.

\subsection{Segmento de Jalisco}

En la Figura 6 se muestran los perfiles que corresponden al segmento de Jalisco en la placa de Rivera. En el perfil F-F' (Figura 6a) se observa fallamiento inverso en la zona de subducción hasta $90 \mathrm{~km}$ de distancia de la trinchera, a una profundidad máxima de aproximadamente $25 \mathrm{~km}$ (evento 124P). Se observa fallamiento normal consistente con la geometría de la placa subducente a una profundidad de 50km (evento 67P). En el Perfil G-G'(Figura 6b) el fallamiento inverso más profundo se encuentra a una profundidad aproximada de $40 \mathrm{~km}$ (evento 92P). No se observa fallamiento normal consistente con la geometría de la placa subducente. El Perfil H-H' (Figura 6c) contiene relativamente pocos eventos y muestra mecanismos inversos a profundidades menores a $20 \mathrm{~km}$, aunque el evento $91 \mathrm{C}$ es inconsistente con estos mecanismos. El evento $50 \mathrm{M}$ a aproximadamente $40 \mathrm{~km}$ de profundidad representa fallamiento normal dentro de la placa subducente. En el perfil I-I' (Figura 6d) el evento más profundo de fallamiento inverso (evento 126C) se encuentra a $33 \mathrm{~km}$ de profundidad. No se observan mecanismos focales con fallamiento normal dentro de la placa subducente.

\section{Zona Sismogénica Interplaca}

Para estimar la profundidad máxima sismogénica en la zona de subducción de Michoacán-Colima-Jalisco se combinaron los perfiles de cada segmento en un solo cuadro (Figura 7), graficando solamente los mecanismos inversos consistentes con deslizamiento en el contacto interplaca y los mecanismos normales. Para el segmento de Michoacán (Figura 7a) se observan eventos de fallamiento inverso hasta una profundidad máxima de $40 \mathrm{~km}$ (eventos $50 \mathrm{C}$ y 78C). No se observan mecanismos de fallamiento normal a profundidades menores a $54 \mathrm{~km}$ con excepción del 

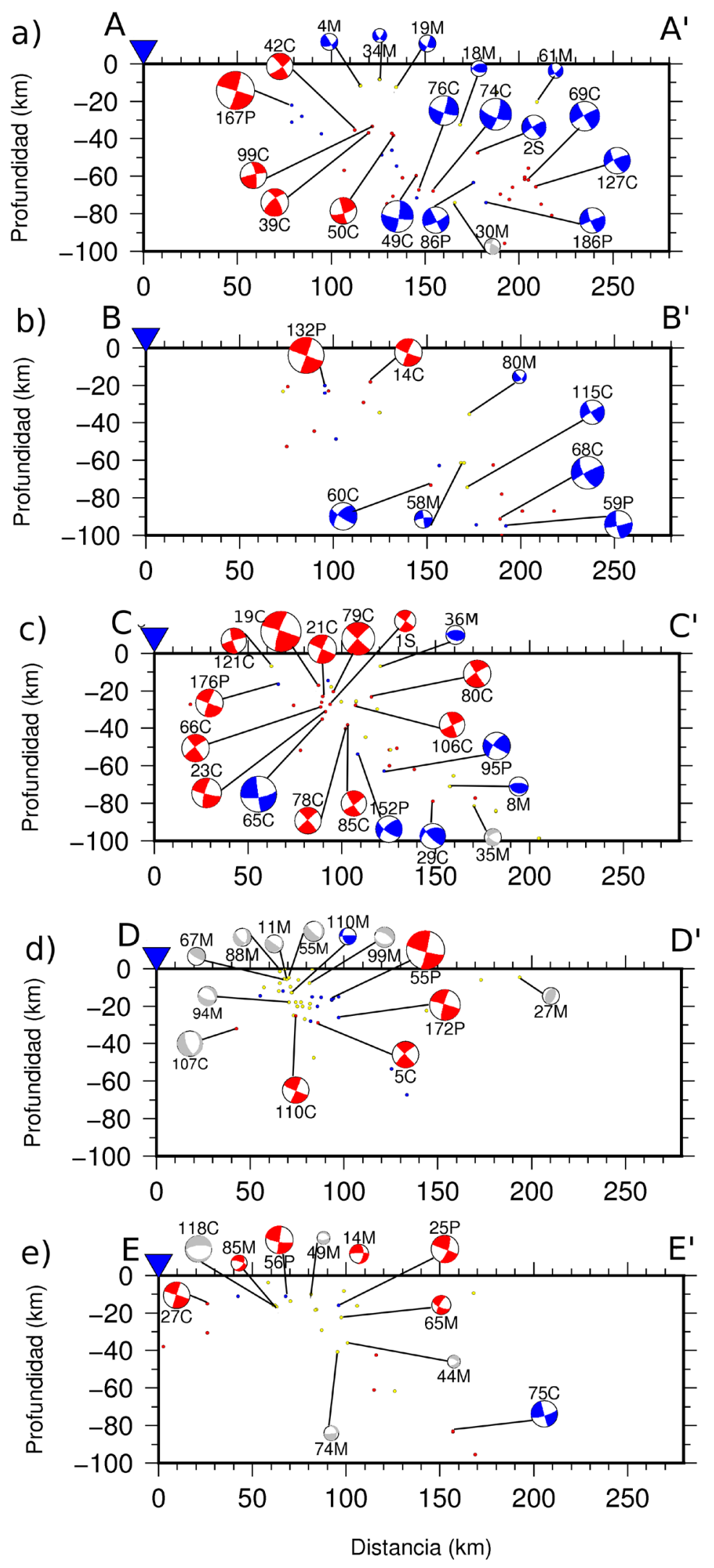

Figura 5. Perfiles A-A'(a), B-B' (b), C-C' (c), D-D'(d) y E-E'(e) de la Figura 4 para el segmento de Michoacán. El triángulo invertido indica la posición de la trinchera. Se muestra la proyección lateral de los mecanismos focales con las etiquetas indicadas en las figuras 2 y 3 . Los mecanismos rojos indican fallamiento inverso consistente con deslizamiento interplaca, los azules indican fallamiento normal y los grises son de fallamiento transcurrente e inverso inconsistentes con deslizamiento en el contacto interplaca. 

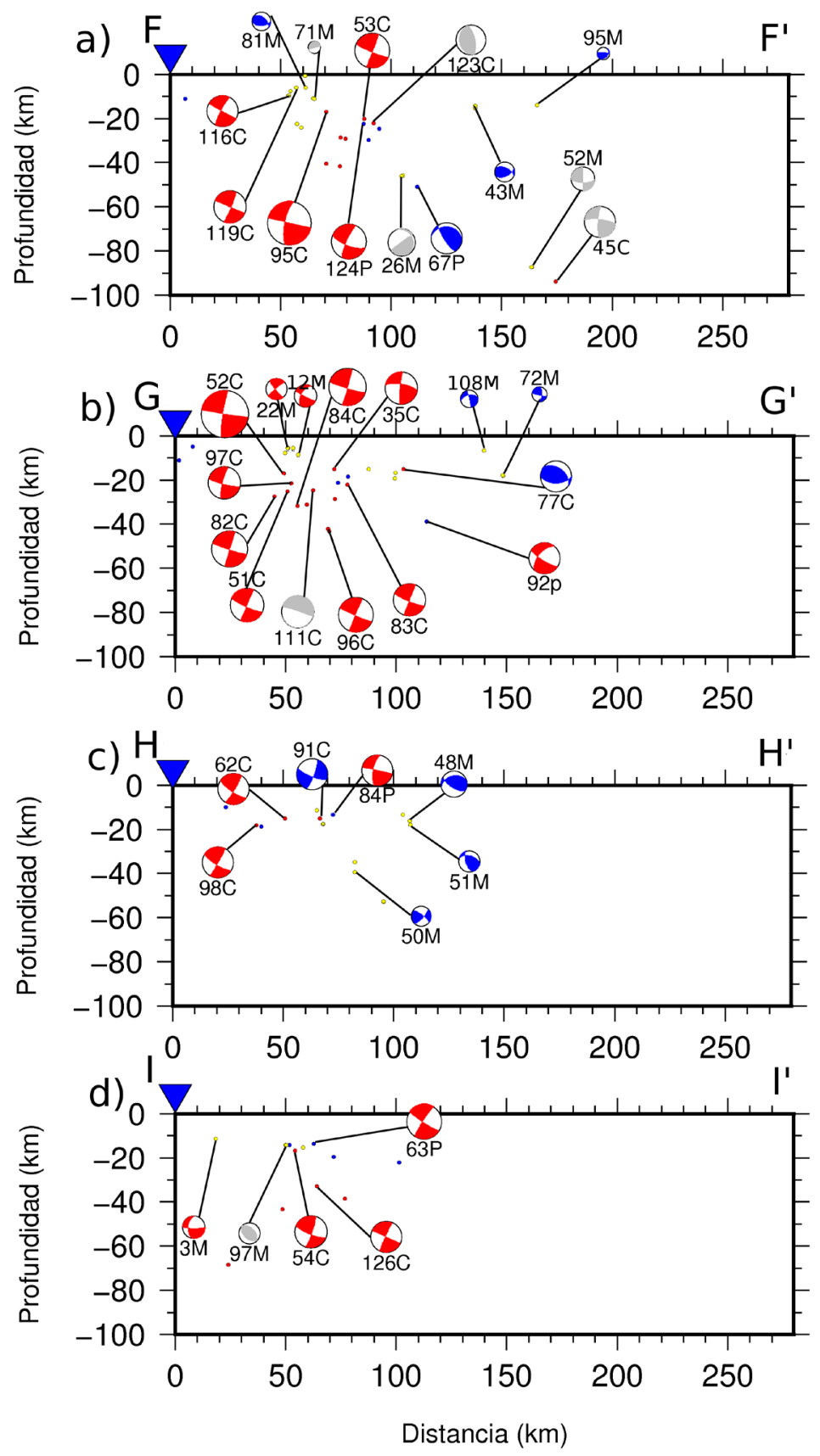

Figura 6. Perfiles F-F'(a), G-G' (b), H-H' (c), y I-I'(d) de la Figura 4 para la región de Colima-Jalisco. Los símbolos son los mismos de la Figura 2 y 3. Los mecanismos rojos indican fallamiento inverso consistente con deslizamiento interplaca, los azules indican fallamiento normal y los grises son de fallamiento transcurrente e inverso inconsistentes con deslizamiento en el contacto interplaca.

evento 65C que ocurrió debajo del sismo Mw 8.0 de 1985. Concluimos que la profundidad del contacto sismogénico en esta porción de la zona de subducción se extiende hasta un mínimo de $40 \mathrm{~km}$.

Este resultado es consistente con las zonas sismogénicas interplaca observadas en otras partes del mundo (Tichelaar y Ruff, 1993; Pacheco et al., 1993) y sugiere una profundidad máxima mayor a la de $25 \mathrm{~km}$ reportada por otros autores para la placa de Cocos (Tichelaar y Ruff, 1993; Pardo y Suárez, 1995; Suárez y Sánchez, 1996). Esta diferencia en los resultados se debe a la clara identificación en este estudio de mecanismos focales de fallamiento inverso hasta profundidades de $40 \mathrm{~km}$ en el segmento de Michoacán que no se habían considerado anteriormente. Tomando esta profundidad máxima podemos estimar un ancho de $95 \mathrm{~km}$ para la zona sismogénica en el segmento de Michoacán 

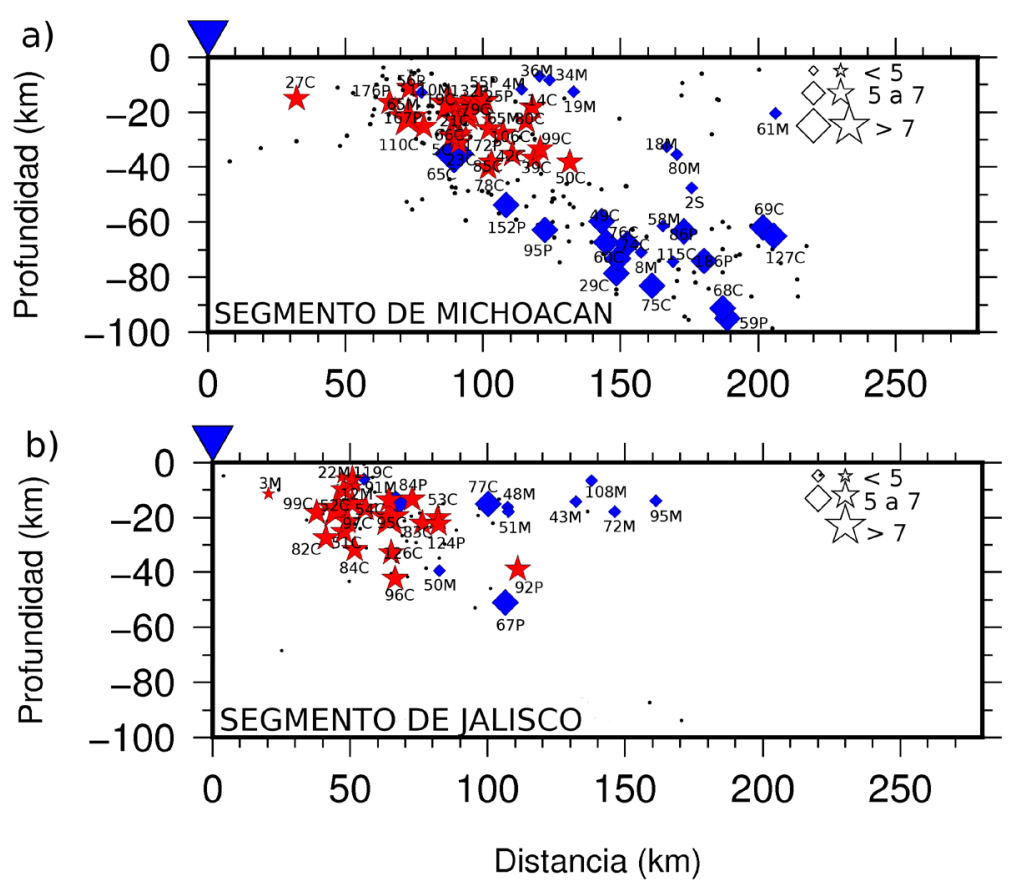

Figura 7. Combinación de los perfiles A, B, C, D y E para el segmento de Michoacan (a) y los perfiles F, G, H, e I para el segmento de Jalisco (b). Las estrellas representan los eventos de fallamiento inverso consistentes con deslizamiento en el contacto interplaca y los diamantes representan los eventos de fallamiento normal. Los círculos negros son los hipocentros que se examinaron en este trabajo.

asumiendo un ángulo de buzamiento de $25^{\circ}$. Este ángulo corresponde al buzamiento propuesto por Pardo y Suárez (1995) para la placa de Cocos y es consistente con el buzamiento promedio de los planos de falla observados para los mecanismos inversos someros en el segmento de Michoacán.

Cabe mencionar que las réplicas de sismos mayores que han ocurrido en el segmento de Michoacán no sobrepasan los $30 \mathrm{~km}$ de profundidad, incluyendo los eventos de septiembre de 1985 (UNAM Seismology Group, 1986) y de Petatlán de 1979 (Valdés et al., 1982). Sin embargo, modelos de deslizamiento determinados para el sismo del 19 de septiembre de 1985 (Mw 8.0) y el sismo de Petatlán de 1979 (Mw 7.4) tienen un ancho de ruptura de aproximadamente 90 km (Mendoza y Hartzell, 1989; Mendoza, 1995), indicando que la profundidad máxima de las réplicas no coincide con el límite del contacto sismogénico. Incluso, trabajos anteriores (p. ej., Mendoza y Hartzell, 1988; Das y Henry, 2003) indican que las áreas de réplicas de los eventos principales no corresponden a las dimensiones de la ruptura cosísmica.

Para el segmento de Jalisco también se combinaron los cuatro perfiles correspondientes a la placa de Rivera en un solo cuadro (Figura 7b). Se observa que el evento más profundo de fallamiento inverso está a una profundidad aproximada de $40 \mathrm{~km}$ (evento 92P). Este evento es el mismo que tomó Pardo y Suárez (1995) para estimar la profundidad máxima del contacto sismogénico en el segmento de Jalisco. El evento $50 \mathrm{M}$ de fallamiento normal está a esta misma profundidad aunque corresponde a un sismo menor $\left(\mathrm{M}_{\mathrm{L}}\right.$ 3.3). No encontramos entonces información adicional que modifique el valor de $40 \mathrm{~km}$ estimado por Pardo y Suárez (1995) para la profundidad máxima del acoplamiento sismogénico. Basado en estos resultados, Pardo y Suárez (1995) sugieren un ancho de $75 \mathrm{~km}$ para la zona sismogénica en el segmento de Jalisco tomando un ángulo de $32^{\circ}$ para la placa de Rivera.

\section{Implicaciones para la Ruptura Sísmica}

De acuerdo a Hanks y Kanamori (1979), el momento sísmico liberado durante un sismo esta definido por el producto de la rigidez, el área de ruptura y el deslizamiento promedio. El momento sísmico entonces aumenta con el área de ruptura, y el tamaño de los sismos en un segmento dado está controlado por el ancho y longitud de ruptura (Heuret et al., 2011). Nuestros resultados entonces tienen implicaciones importantes para la estimación de la magnitud máxima esperada para sismos mayores en la zona de subducción de Michoacán-Colima-Jalisco.

La historia sísmica reciente en el segmento de Michoacán indica que el deslizamiento sísmico principal ha ocurrido en secciones (ver Figura 1), relacionado probablemente a la existencia de asperezas en el contacto interplaca (Mendoza, 1993). Sin embargo, existen zonas de subducción a nivel mundial donde se han observado secuencias de rupturas adyacentes durante una época, alternadas con la ocurrencia 
de un solo megasismo en otra época durante diferentes ciclos sísmicos. En estos casos, los megasismos incorporan las zonas de ruptura de los sismos anteriores, resultando en un comportamiento variable de ruptura caracterizado por una repetición de deslizamiento en zonas específicas a lo largo del contacto interplaca. Ejemplos incluyen las zonas de subducción de Colombia-Ecuador (Kelleher, 1972; Kanamori y McNally, 1982), Nankai en Japón (Imamura, 1928; Ando, 1975), y Sumatra-Andaman en Indonesia (Bilham et al., 2005; Kanamori, 2006). El megasismo del 26 de diciembre del 2004 de Sumatra-Andaman (Mw 9.2), ocurrió en una zona donde no existían registros históricos e instrumentales de sismos con magnitud mayor a 8. El sismo rompió una longitud de $1400 \mathrm{~km}$ que abarcó las zonas de ruptura de los sismos mayores generados anteriormente en la zona.

Este comportamiento de ruptura variable sugiere la posibilidad de que todo el segmento de Michoacán se deslice en una sola ruptura sísmica. En la región de Oaxaca en México, por ejemplo, se tienen reportes históricos de un sismo de una magnitud estimada de Mw 8.6 que ocurrió el 28 de marzo de 1787 con una longitud de ruptura de $450 \mathrm{~km}$, aunque en los últimos 100 años se han generado sismos de magnitudes de entre 7.3 a 8.2 (Suárez y Albini, 2009). Existen leyes de escalamiento tanto empíricas (p. ej., Wells y Coppersmith, 1994) como teóricas (p. ej., Leonard, 2010) que relacionan los parámetros de la ruptura, como las dimensiones y el deslizamiento, al momento sísmico. Algunas de estas relaciones se han desarrollado específicamente para zonas de subducción (Somerville et al., 2002; Murotani et al., 2008; Blaser et al., 2010; Strasser et al., 2010; Ramírez-Gaytán et al., 2014).

Ramírez-Gaytán et al. (2014) derivaron relaciones empíricas de escalamiento para la zona de subducción de México a partir de modelos de ruptura determinados para siete sismos mayores o igual a MW 6.9 ocurridos de 1979 a 2003 y ubicados principalmente en la región de Guerrero-Colima-Michoacán. Sin embargo, las relaciones de Ramírez-Gaytán et al. (2014) subestiman la magnitud del evento del 19 de septiembre $1985 \mathrm{si}$ se asumen dimensiones consistentes con el modelo de ruptura determinado por Mendoza y Hartzell (1989). Estas relaciones también subestiman la magnitud obtenida por Singh et al. (1984) para el sismo de Jalisco del 3 de junio 1932 si se utilizan las dimensiones del área de réplicas para estimar la magnitud. En este trabajo preferimos utilizar las leyes de escalamiento de Murotani et al. (2008), las cuales están basadas en eventos de subducción en Japón, porque producen valores más apropiados para estos dos eventos. Cabe indicar, sin embargo, que las leyes de Murotani et al. (2008) no consideraron el megasismo de Tohoku, Japón de 2011 (Mw 9.0) por lo que podrían subestimar la magnitud para eventos mayores a Mw 8.

Si consideramos un área $\mathrm{A}\left(\mathrm{en} \mathrm{km}^{2}\right)$ que corresponde a una longitud de $300 \mathrm{~km}$ y un ancho de $95 \mathrm{~km}$ para el segmento de Michoacán, la relación de Murotani et al.
(2008)

$$
\mathrm{A}=1.48 \times 10^{-10} \mathrm{Mo}^{2 / 3}
$$

indicaría un momento sísmico Mo de $2.7 \times 10^{21}$ newtonmetros (nt-m), que corresponde a una magnitud $\mathrm{Mw}$ de 8.25. La ecuación de Murotani et al. (2008) que relaciona el deslizamiento promedio $\mathrm{D}$ (en $\mathrm{m}$ ) y el momento sísmico (en nt-m)

$$
\mathrm{D}=1.48 \times 10^{-7} \mathrm{Mo}^{1 / 3}
$$

indicaría un deslizamiento de $2 \mathrm{~m}$ a lo largo del segmento. Cabe mencionar que el deslizamiento máximo para el sismo podría ser mucho mayor a $2 \mathrm{~m}$, tomando en cuenta que la distribución de deslizamiento a lo largo de una falla es generalmente heterogéneo e involucra la fractura de asperezas independientes en el plano del contacto interplaca.

En lo que respecta a la placa de Rivera en la región de Colima-Jalisco, es difícil saber la longitud máxima que podría tener un sismo mayor pero si el área de réplicas de los sismos de 1932 define el límite norte del evento, entonces este se podría extender por una distancia de aproximadamente $420 \mathrm{~km}$. Aplicando la ecuación 1 considerando un ancho máximo de $75 \mathrm{~km}$ para la ruptura podemos estimar un momento sísmico de $3.1 \times 10^{21}$ nt$\mathrm{m}$, indicando una magnitud de $8.3 \mathrm{Mw}$ comparable a la magnitud máxima estimada para el segmento de Michoacán. Esta magnitud es mayor a la magnitud máxima de Mw 8.15 estimada por Singh et al. (1985) en base a la edad de la placa de Rivera y la velocidad de convergencia. Estimaciones de magnitud máxima basadas en las edades de las placas se han cuestionado recientemente porque no coinciden con los tamaños de los megasismos ocurridos en los últimos años en Indonesia 2004 y Japón 2011 (Stein y Okal, 2011).

No se puede descartar la posibilidad de que una sola ruptura en un megasismo incluya los dos segmentos de Michoacán y de Jalisco. Sin embargo, esto es quizás poco probable ya que ambos segmentos pertenecen a placas tectónicas distintas con diferentes geometrías de la zona de contacto interplaca. Sería más probable que una ruptura en el segmento de Michoacán se extienda al sur más allá de la zona de fracturas de Orozco. En ese caso, el tamaño del sismo esperado sería mayor a la magnitud de Mw 8.25 estimada en este trabajo para el segmento de Michoacán.

La historia sísmica en la zona de subducción de Michoacán-Colima-Jalisco indica que los segmentos de Michoacán y Jalisco se han roto principalmente en eventos de magnitud entre 7.2 y 8.1 durante el último siglo. El proceso de ruptura durante estos sismos mayores puede ser complejo donde se pueden romper más de una aspereza. Entre cada aspereza pueden quedar espacios en el contacto interplaca donde se podría generar otro sismo a futuro, asumiendo que esas zonas no se han deslizado anteriormente. Por ejemplo, el sismo del 25 de octubre de 1981 ocurrió entre dos asperezas que se rompieron 4 años 
después durante el evento del 19 de septiembre de 1985 (Mendoza, 1993). Es difícil saber con anticipación el lugar y el tamaño de los sismos que se podrían generar en el contacto interplaca. Sin embargo, nuestros resultados identifican áreas donde podría haber un deslizamiento cosísmico futuro, asumiendo que los sismos continúan rompiendo secciones parciales de ambos segmentos.

Sería importante entonces examinar a detalle la distribución de las rupturas históricas para tratar de entender el proceso de generación de sismos mayores en la zona de subducción Michoacán-Colima-Jalisco. Este historial aportaría conocimientos importantes que ayudarían a identificar los posibles tiempos de recurrencia y el potencial sísmico. La ubicación e interacción de las asperezas responsables por la generación de los sismos mayores podrían identificar áreas en la zona de contacto interplaca predispuestas a experimentar deslizamiento cosísmico en el futuro cercano. El estudio detallado de la distribución de las rupturas históricas en los segmentos de Michoacán y Jalisco está fuera del alcance de este trabajo que se concentra principalmente en la estimación del ancho del contacto sismogénico basado en la ubicación de los mecanismos inversos observados.

\section{Conclusiones}

Los hipocentros y mecanismos focales, observados entre 1964 y 2008 en la zona de subducción de MichoacánColima-Jalisco, nos permitieron examinar el ancho de la zona de acoplamiento sismogénico del segmento de Michoacán en la placa de Cocos y el segmento de Jalisco en la placa de Rivera. A partir de un análisis detallado de perfiles de sismicidad perpendicular a la trinchera Mesoamericana encontramos que la profundidad máxima de la zona sismogénica en ambos segmentos es aproximadamente $40 \mathrm{~km}$.

Para el segmento de Michoacán, esta profundidad máxima contrasta con los $\sim 25 \mathrm{~km}$ propuestos anteriormente por varios autores para toda la placa de Cocos en México (Tichelaar y Ruff, 1993; Pardo y Suárez, 1995; Suárez y Sánchez, 1996). Nuestro análisis incluye mecanismos focales inversos que no estaban disponibles anteriormente y que sugieren una profundidad máxima consistente con los valores de 40 - $55 \mathrm{~km}$ observados en otras partes del mundo. Esto indica que la porción noroeste de la zona de subducción de la placa de Cocos no es anómala. Nuestro estudio considera únicamente la sección que pertenece al segmento de Michoacán y no podemos especular sobre la profundidad del contacto sismogénico al sureste de la zona de fractura de Orozco.

La profundidad máxima de $40 \mathrm{~km}$ observada para eventos inversos consistentes con deslizamiento en el contacto interplaca en el segmento de Michoacán sugiere un ancho sismogénico de aproximadamente $95 \mathrm{~km}$ tomando un ángulo de buzamiento de $25^{\circ}$ para la porción noroeste de la placa de Cocos. Este ancho de $95 \mathrm{~km}$ es consistente con las dimensiones de las rupturas determinadas a partir de la modelación de las formas de onda registradas para varios sismos recientes de $\mathrm{Mw}>7$ en el segmento de Michoacán, incluyendo el sismo del 19 de septiembre de 1985 (Mw 8.0) y el sismo de Petatlán de 1979 (Mw 7.4). Para la placa de Rivera, no existen mecanismos inversos adicionales que modifiquen la profundidad máxima de $40 \mathrm{~km}$ estimada por Pardo y Suárez (1995) para el acoplamiento sismogénico. Pardo y Suárez (1995) determinan un ancho de $75 \mathrm{~km}$ para la zona sismogénica asumiendo un ángulo de buzamiento de $32^{\circ}$. La longitud del segmento de Michoacán $(300 \mathrm{~km})$ es menor a la longitud del segmento de Jalisco $(420 \mathrm{~km})$ pero el mayor ancho del contacto sismogénico en Michoacán indica un área similar a la del segmento de Jalisco, sugiriendo un valor similar (Mw 8.3) para la magnitud máxima esperada en ambos segmentos y un deslizamiento promedio de $\sim 2 \mathrm{~m}$.

\section{Agradecimientos}

Este trabajo fue financiado por el proyecto UNAM/ PAPIIT IN104013. Se agradece al Conacyt por el apoyo otorgado a M. R. Martínez López. Las imágenes se realizaron con el programa GMT (Generic Mapping Tools) de Wessel y Smith (1991). Agradecemos además los comentarios del editor D. Legrand y de los revisores B. Delouis y G. Suárez que mejoraron la presentación del trabajo.

\section{Referencias}

Anderson, J.G., Singh, S.K., Espíndola, J.M., Yamamoto, J., 1989, Seismic strain release in the Mexican subduction thrust: Physics of the Earth and Planet Interiors, 58, 307-332.

Ando, M., 1975, Source mechanisms and tectonic significance of historical earthquakes along the Nankai Trough, Japan: Tectonophysics, 27, 119-140.

Bandy, W.L., Mortera-Gutiérrez, C., Urrutia-Fucugauchi, J., Hilde, T.W.C., 1995, The subducted Rivera-Cocos plate boundary: Where is it, what is it, and what is its relationship to the Colima rift?: Geophysical Research Letters, 22, 3075-3078.

Bandy, W.L., Kostoglodov, V., Mortera-Gutiérrez C.A., 1998, Southwest migration of the Instantaneous Rivera-Pacific Euler pole Since 0.78 Ma: Geofísica Internacional, 37, 153-169.

Bilham, R., Engdahl, R., Feldl, N., Satyabla, S.P., 2005, Partial and complete rupture of the Indo-Andaman plate boundary: Seismological Research Letters, 76, 299-311.

Blaser, L., Krüger, F., Ohrnberger, M., Scherbaum, F., 2010, Scaling relations of earthquake source parameter estimates with special focus on subduction environment: Bulletin of the Seismological Society of America, 100, 2914-2926.

CMT, 2013, Global CMT Project, disponible en http://www.globalcmt.org/ CMTsearch.html, actualizado el 30 de marzo de 2013, consultado en abril de 2014.

Courboulex, F., Singh, S.K., Pacheco, J.F., 1997, The 1995 Colima-Jalisco, Mexico, earthquake $(\mathrm{Mw}=8)$ : a study of the rupture process: Geophysical Research Letters, 24, 1019-1022.

Currie, C.A., Hyndman, R.D., Wang, K., Kostoglodov, V., 2002, Thermal models of the Mexico subduction zone: Implications for the 
megathrust seismogenic zone: Journal of Geophysical Research, 107(B12), 2370.

Das, S., Henry, C., 2003, Spatial relation between main earthquake slip and its aftershock distribution: Reviews of Geophysics, 41(3), 1013.

DeMets, C., Stein, S., 1990, Present-day Kinematics of the Rivera Plate and Implications for Tectonics in Southwestern Mexico: Journal of Geophysical Research, 95(B13), 21,931-21,948.

DeMets, C, Wilson, D.S., 1997, Relative motions of the Pacific, Rivera, North American, and Cocos plate since 0.78 Ma: Journal of Geophysical Research, 102(B2), 2789-2806.

Dewey, J.W., 1971, Seismicity studies with the method of joint hypocenter determination, Universidad of California, Berkeley, Tesis doctoral, $163 \mathrm{p}$.

Duda, S.J., 1965, Secular seismic energy release in the circum Pacific belt: Tectonophysics, 2(5), 409-452.

Eissler, H.K., McNally, K.C., 1984, Seismicity and tectonics the Rivera plate and implications for the $1932 \mathrm{Jalisco}$, Mexico, earthquake: Journal of Geophysical Research, 89(B6), 4520-4530.

Eissler, H., Astiz, L., Kanamori, H., 1986, Tectonic Setting and Source Parameters of the September 19, 1985 Michoacan, Mexico Earthquake: Geophysical Research Letters, 13(6), 569-572.

Engdahl, E.R., Vander Hilst, R.D., Buland, R.P., 1998, Global teleseismic earthquake relocation with improved travel times and procedures for depth determination: Bulletin of the Seismological Society of America, 88, 722-743.

Gettrust, J., Hsu, V., Helsley, C.E, Herrero, E., Jordan, T., 1981, Pattern of local seismicity preceding Petatlan earthquake of 14 March 1979: Bulletin of the Seismological Society America, 71, 761-769.

Gutenberg, B., Richter C.F., 1954, Seismicity of the Earth and Associated Phenomena, 2da edition, Princeton University Press, Princeton, New Jersey, 310.

Hanks, T.C., Kanamori H., 1979, Moment magnitude scale: Journal of Geophysical Research, 84, 2348-2350.

Havskov, J., Singh, S.K., Nava, E., Dominguez, T., Rodríguez, M., 1983, Playa Azul, Michoacán, Mexico Earthquake of 25 October 1981 (Ms =7.3): Bulletin of the Seismological Society America, 73, 449-457.

Heuret, A., Lallemand, S., Funiciello, F., Piromallo, C., Faccenna, C., 2011, Physical characteristics of subduction interface type seismogenic zones revisited: Geochemistry Geophysics Geosystems, 12(1), Q01004.

Imamura, A., 1928, On the seismic activity of central Japan: Japanese Journal of Astronomy and Geophysics, 6, 119-137.

ISC (2009), International Seismological Centre, EHB Bulletin, http://www. isc.ac.uk, Thatcham, United Kingdom, consultado en noviembre de 2012.

Kanamori, H., 2006, Lessons from the 2004 Sumatra-Andaman earthquake: Philosophical Transactions of the Royal Society A, 364, 1927-1945.

Kanamori, H., McNally, K.C., 1982, Variable rupture mode of the subduction zone along the Ecuador-Colombia coast: Bulletin of the Seismological Society America, 72, 1241-1253.

Kelleher, J.A., 1972, Rupture zones of large South America earthquakes and some predictions: Journal of Geophysical Research, 77(11), 2087-2103.

Kelleher, J., Sykes, L., Oliver, J., 1973, Possible criteria for predicting earthquake locations and their application to major plate boundaries of the Pacific and Caribbean: Journal of Geophysical Research, 78(14), 2547- 2585.

Larson, L.R., 1972, Bathymetry, Magnetic Anomalies and Plate History of the Mouth of the Gulf of California: The Geological Society of America Bulletin, 83(11), 3345-3360.

Leonard, M., 2010, Earthquake Fault Scaling: Self-Consistent Relating of Rupture Length, Width, Average Displacement, and Moment Release: Bulletin of the Seismological Society of America, 100(5A), 1971-1988.

Martínez-López, R., 2011, Estudio Sísmico de la Estructura Cortical en el Bloque de Jalisco a Partir de Registros Locales del Proyecto MARS, Juriquilla Querétaro, México, Centro de Geociencias, Universidad Nacional Autónoma de México (UNAM), Tesis de Maestría, 129 p.
Mendoza, C., 1993, Coseismic slip to two large Mexican earthquakes From teleseismic body waveforms Implications for asperity interaction in the Michoacán Plate Boundary Segment: Journal of Geophysical Research, 98(B5), 8197- 8210.

Mendoza, C., 1995, Finite-Fault Analysis of the 1979 March 14 Petatlan, Mexico, Earthquake Using Teleseismic P-Wave-Forms: Geophysical Journal International, 121(3), 675-683.

Mendoza, C., Hartzell, S., 1988, Aftershock patterns and main shock faulting, Bulletin of the Seismological Society America: 78, 14381449.

Mendoza, C., Hartzell, S., 1989, Slip distribution of the 19 September 1985 Michoacan, Mexico: Bulletin of the Seismological Society America, 79(3), 655-669.

Mendoza, C., Hartzell S., 1999, Fault-Slip Distribution of the 1995 ColimaJalisco, Mexico, Earthquake: Bulletin of the Seismological Society America, 89(5), 1338-1344.

Murotani, S., Miyake, H., Koketsu, K., 2008, Scaling of characterized slip models for plate-boundary earthquakes: Earth Planet Space, $60,987-991$

Nixon, G.T., 1982, The relationship between Quaternary volcanism in central Mexico and the seismicity and the structure of subducted ocean lithosphere: Geological Society America Bulletin, 93(6), 514-523.

Ohta, Y., Miura, S., Ohzono, M., Kita, S., Iinuma, T., Demachi, T., Tachibana, K., Nakayama, T., Hirahara, S., Suzuki, S., Sato, T., Uchida, S., Hasegawa, A., Umino, N., 2011, Large intraslab earthquake (2011 April 7, M7.1) after the 2011 off the Pacific coast of Tohoku Earthquake (M9.0): Coseismic fault modelbased on the dense GPS network data: Earth Planets Space, 63, 1207-1211.

Pacheco, J., Sykes, L.R., Scholz, C.H., 1993, Nature of seismic coupling of the subduction type: Journal of Geophysical Research, 98, 14133-14159.

Pardo, M., 1993, Características sismotectónicas de la subducción de las placas de Rivera y Cocos en el sur de México, D.F., México, Universidad Nacional Autónoma de México (UNAM), Instituto de Geofísica, Tesis doctoral, 128p.

Pardo, M., Suárez, G., 1995, Shape of the subducted Rivera and Cocos plates in southern Mexico: Seismic and tectonic implications: Journal of Geophysical Research, 100(B7), 12357-12373.

Peláez-Gaviria, J.R., Mortera-Gutiérrez, C.A., Bandy, W.L., Michaud, F., 2013, Morphology and magnetic survey of the Rivera-Cocos plate boundary of Colima, Mexico: Geofísica Internacional, 52(1), 73-85.

Priestley, K.F., Masters T.G., 1986, Source mechanism of the September 19, 1985 Michoacan earthquake and its implications, Geophysical Research Letters, 13, 601-604.

Ramírez-Gaytán, A., Aguirre, J., Jaimes, M.A., Huérfano, V., 2014, Scaling Relationships of source Parameters of Mw 6.9-8.1 Earthquakes in the Cocos-Rivera-North American Subduction Zone: Bulletin of the Seismological Society America, 104(2), 840-854.

Ramírez-Herrera, M.T., Kostoglodov, V., Urrutia-Fucugauchi, J., 2010, Overview of Recent Coastal Tectonic Deformation in the Mexican Subduction Zone: Pure and Applied Geophysics, 168(8), 1425-1433.

Reyes, A., Brune, J.N., Lomnitz C., 1979, Source mechanism and aftershock study of the Colima, México, earthquake of 10 January, 1973: Bulletin of the Seismological Society America, 69, 1819-1840.

Santoyo, A.M., Singh S.K., Mikumo T., 2005, Source process and stress change associated with the 11 January, $1997(\mathrm{Mw}=7.1)$ Michoacán, Mexico, inslab earthquake: Geofísica Internacional, 44, 4, 317-330.

Santoyo, A.M., Mikumo, T., Quintanar, L., 2006, Faulting process and coseismic stress change during the 30 January, 1973, Colima, Mexico interpolate earthquake $(\mathrm{Mw}=7.6)$ : Geofisica Internacional, 45(3), 163-178.

Serrato-Díaz, G., Bandy, W.L., Mortera-Gutiérrez C.A., 2004, Active rifting and crustal thinning along the Rivera-Cocos plate boundary as inferred from Mantle Bouguer gravity anomalies: Geofísica Internacional, 43, 361-381.

Singh, S.K., Astiz, L., Havskov, J., 1981, Seismic Gaps and Recurrence Periods of large Earthquakes along the Mexican Subduction zone a reexamination: Bulletin of the Seismological Society America, 
$71(3), 827-843$.

Singh, S.K., Domínguez, T., Castro, R., Rodríguez, M., 1984, P waveform of large shallow earthquake along the Mexican subduction zone: Bulletin of the Seismological Society America, 74(6), 2135-2156.

Singh, S.K., Ponce, L., Nishenko, S.P., 1985, The great Jalisco, Mexico, earthquakes of 1932: Subduction of the Rivera Plate: Bulletin of the Seismological Society America, 75(5), 1301-1313.

Snoke, J.A., Munsey, J.W., Teague, A.C., Bollinger, G.A., 1984, A program for focal mechanism determination by combined use of polarity of SV-P amplitude ratio data: Earthquake Notes 55(3), 15-20.

SSN, 2013, Servicio Sismológico Nacional, disponible en la página de $\mathrm{http}: / /$ laxdoru.igeofcu.unam. $\mathrm{mx} / \sim \mathrm{cmt} /$, consultado en agosto de 2013.

Somerville, P., Collins, N., Sato, T., Ishii, T., Dan, K., Fujiwara, H., 2002, Characterizing heterogeneous slip models for large subduction earthquakes for strong ground motion prediction, en Proceedings of the $11^{\text {th }}$ Japan Earthquake Engineering Symposium, 1, 163-166 (in Japanese).

Stein, S., Okal, E., 2011, The size of the 2011 Tohoku Earthquake Need Not Have Been a Surprise: EOS, 92(27), 227-228.

Strasser, F.O., Arango, M.C., Bommer, J.J., 2010, Scaling of the source dimensions of interface and intraslab subduction-zone earthquakes with moment magnitude: Seismological Research Letters, 81, 941-950.

Suárez, G., Albini, P., 2009, Evidence for Great Tsunamigenic Earthquakes (M 8.6) along the Mexican Subduction Zone: Bulletin of the Seismological Society America, 99(2A), 892-896.

Suárez, G., Comte, D., 1993, Comment on "Seismic coupling along the Chilean subduction zone" by B. W. Tichelaar and L.R. Ruff: Journal of Geophysical Research, 98, 15,825-15,828.

Suárez, G., Sánchez, O., 1996, Shallow depth of seismogenic coupling in southern Mexico: implications for the maximum size of earthquakes in the subduction zone: Physics of the Earth Planetary Interiors, 93, 53-61.
Suárez, G., Monfret, T., Wittlinger, G., David, C., 1990, Geometry of subduction and depth of the seismogenic zone in the Guerrero Gap, México: Nature, 345, 336-338.

Tichelaar, B.W., Ruff, L.J., 1993, Depth of seismic coupling along subduction zones: Journal of Geophysical Research: Solid Earth, 98(B2), 2017-2037.

UNAM Seismology Group, 1986, The September 1985 Michoacán Earthquakes: Aftershock Distribution and History of Rupture: Geophysical Research Letters, 13(6), 573-576.

UNAM Seismology Group, 2015, Papanoa, Mexico earthquake of 18 April 2014 (Mw 7.3): Geofísica Internacional, 54(4), 363-386.

Valdés, C., Meyer R.P., Zúñiga, R., Havskov, J., Singh, S.K., 1982, Analysis of the Petatlán aftershocks: Numbers, energy release, and asperities: Journal of Geophysical Research: Solid Earth, 87, B10, 8519-8527.

Wells, D.L., Coppersmith, K.J., 1994, New empirical relationships among magnitude, rupture length, rupture width, rupture area, and surface displacement: Bulletin of the Seismological Society America, 84, 974-1002.

Wessel, P., Smith, W.H.F., 1991, Free software helps map and display data, EOS Transactions: American Geophysical Union, 72, 441.

Yagi, Y., Mikumo, T., Pacheco, J., 2004, Source rupture of the Tecoman, Colima, México earthquake of January 22, 2003, determined by joint inversion teleseismic body wave and near field data: Bulletin of the Seismological Society America, 94, 1795-1807.

Yang, T., Grand, S.P., Wilson, D., Guzman-Speziale, M., Gomez-Gonzalez, J.M., Dominguez-Reyes, T., Ni, J., 2009, Seismic structure beneath the Rivera subduction zone from finite-frequency seismic tomography: Journal of Geophysical Research, 114, B01302.

Manuscrito recibido: Agosto 1, 2014

Manuscrito corregido recibido: Junio 19, 2015

Manuscrito aceptado: Junio 25, 2015 